\title{
A transcriptomic signature mediated by HOXA9 promotes human glioblastoma initiation, aggressiveness and resistance to temozolomide
}

\author{
Marta Pojo ${ }^{1,2}$, Céline S. Gonçalves ${ }^{1,2}$, Ana Xavier-Magalhães ${ }^{1,2}$, Ana Isabel Oliveira ${ }^{1,2}$, \\ Tiago Gonçalves ${ }^{1,2}$, Sara Correia ${ }^{3}$, Ana J. Rodrigues ${ }^{1,2}$, Sandra Costa ${ }^{1,2}$, \\ Luísa Pinto ${ }^{1,2}$, Afonso A. Pinto ${ }^{4}$, José M. Lopes ${ }^{5,6,7}$, Rui M. Reis ${ }^{1,2,8}$, Miguel Rocha ${ }^{3}$, \\ Nuno Sousa ${ }^{1,2}$, Bruno M. Costa ${ }^{1,2}$ \\ ${ }^{1}$ Life and Health Sciences Research Institute (ICVS), School of Health Sciences, University of Minho, Campus de Gualtar \\ 4710-057 Braga, Portugal \\ ${ }^{2}$ ICVS/3B's-PT Government Associate Laboratory, Braga/Guimarães, Campus de Gualtar 4710-057 Braga, Portugal \\ ${ }^{3}$ Centre of Biological Engineering/Department of Informatics, University of Minho, Campus de Gualtar 4710-057 Braga, \\ Portugal \\ ${ }^{4}$ Department of Neurosurgery, Hospital de Braga, Sete Fontes, 4710-243 São Victor, Braga, Portugal \\ ${ }^{5}$ Department of Pathology, Hospital S. João, Alameda Professor Hernâni Monteiro, 4200-319 Porto, Portugal \\ ${ }^{6}$ Institute of Molecular Pathology and Immunology at the University of Porto (IPATIMUP), Rua Dr. Roberto Frias s/n $4200-465$ \\ Porto, Portugal \\ ${ }^{7}$ Medical Faculty, University of Porto, Alameda Professor Hernâni Monteiro, 4200-319 Porto, Portugal \\ ${ }^{8}$ Barretos Cancer Hospital, Molecular Oncology Research Center, Rua Antenor Duarte Vilela, 1331-Doutor Paulo Prata, \\ Barretos-SP, 14780-000, Brasil
}

Correspondence to:

Bruno M. Costa, e-mail: bfmcosta@ecsaude.uminho.pt

Keywords: Glioblastoma, prognosis, oncogene, temozolomide, HOXA9

Received: September 22, $2014 \quad$ Accepted: January 16, $2015 \quad$ Published: February 20, 2015

\section{ABSTRACT}

Glioblastoma is the most malignant brain tumor, exhibiting remarkable resistance to treatment. Here we investigated the oncogenic potential of HOXA9 in gliomagenesis, the molecular and cellular mechanisms by which HOXA9 renders glioblastoma more aggressive, and how HOXA9 affects response to chemotherapy and survival. The prognostic value of HOXA9 in glioblastoma patients was validated in two large datasets from TCGA and Rembrandt, where high HOXA9 levels were associated with shorter survival. Transcriptomic analyses identified novel HOXA9-target genes with key roles in cancer-related processes, including cell proliferation, DNA repair, and stem cell maintenance. Functional studies with HOXA9-overexpressing and HOXA9-silenced glioblastoma cell models revealed that HOXA9 promotes cell viability, stemness and invasion, and inhibits apoptosis. Additionally, HOXA9 promoted the malignant transformation of human immortalized astrocytes in an orthotopic in vivo model, and caused tumor-associated death. HOXA9 also mediated resistance to temozolomide treatment in vitro and in vivo via upregulation of BCL2. Importantly, the pharmacological inhibition of BCL2 with the BH3 mimetic ABT-737 reverted temozolomide resistance in HOXA9-positive cells. These data establish HOXA9 as a driver of glioma initiation, aggressiveness and resistance to therapy. In the future, the combination of $\mathrm{BH} 3$ mimetics with temozolomide should be further explored as an alternative treatment for glioblastoma. 


\section{INTRODUCTION}

Glioblastoma (GBM) is the most common and malignant glioma type of the central nervous system [1]. Annually $\sim 10,000$ new cases are diagnosed in the United States, and $>50,000$ patients live with the disease [2]. The clinical evolution is poor and variable among patients [3], and $~ 32 \%$ of all diagnosed cases survive less than 1 year [2]. A pivotal phase III clinical trial in 2005 showed that temozolomide-based chemotherapy plus radiation was significantly more effective than radiation alone [4]. A more recent study validated the therapeutic benefit of radiotherapy with concomitant and adjuvant temozolomide as compared to radiotherapy alone [5]. The methylation status of the promoter region of $M G M T$ is one of the most promising prognostic biomarkers of GBM: a methylated $M G M T$ promoter is associated with a more effective tumor response to temozolomide and increased survival of GBM patients [6]. Mechanistically, MGMT methylation reduces gene expression, which decreases tumor cells' ability to repair temozolomide-induced DNA damages, thus increasing drug sensitivity [6]. However, this association is not universal, as some tumors with a methylated MGMT promoter do not benefit from temozolomide treatment, while others with unmethylated $M G M T$ respond favorably [7]. Therefore, there is an emerging need in discovering new molecular markers of drug response.

An aberrant expression of many of the $39 \mathrm{HOX}$ genes has been found in various human cancers, affecting several hallmarks of cancer, including increased proliferation, angiogenesis, invasion, and resistance to apoptosis [8-11]. In gliomas, several $H O X$ genes were shown to be part of large gene expression signatures that are associated with the maintenance of GBM stem cells and therapy resistance [12-15]. Specifically, it was shown that HOXA9 and HOXA10 have prognostic value in adult and pediatric high-grade glioma patients $[12,13$, 16]. While HOXA10 was recently shown to drive the expression of genes with critical roles in gliomagenesis [14] and to increase temozolomide resistance in vitro [15], the downstream mechanisms by which HOXA9 may contribute to poor outcomes in GBM patients have not been addressed. Given its prognostic value, a more complete understanding of the molecular targets and the functional consequences of HOXA9 activation on the establishment and maintenance of the malignant phenotype of glioblastoma is required.

In this report, we pinpoint the genome-wide transcriptome of HOXA9 in GBM and demonstrate its functional relevance in initiating gliomas in vivo using immortalized astrocytes and established GBM cells. We also present data showing that HOXA9 promotes several oncogenic features, including increased cell viability, invasion, and stem cell-like features, and decreased sensitivity to temozolomide treatment in vitro and in vivo, and identify BCL2 as a putative therapeutic target to improve the response of HOXA9-positive GBMs to temozolomide. Our findings provide new insights regarding strategies to target HOXA9 overexpression in this incurable cancer.

\section{RESULTS}

\section{HOXA9 is overexpressed and has prognostic value in GBM patients}

HOXA9 expression was analyzed in WHO grades II/III glioma patients (27) and grade IV GBM patients (572) deposited in TCGA [17]. HOXA9 was found to be highly overexpressed in a subset of GBM patients comparing to lower grades glioma (LGG, WHO grades II/ III) patients and normal controls (Figure 1A), confirming that HOXA9 is associated with glioma grade and may be important in tumor progression. According to the four GBM molecular subgroups [18], HOXA9 overexpression was more frequent in the mesenchymal $(10.34 \%)$ and in the proneural subtypes $(7.02 \%$; Figure 1B).

In order to explore putative mechanisms responsible for HOXA9 overexpression in GBM, HOXA9 copy number aberrations and DNA methylation levels were evaluated using the TCGA database (Figure 1C and 1D). HOXA9 was found to be amplified in $31 \%$ (114/372) of GBM patients (Figure 1C), of which only 7.9\% (9/114) presented high levels of HOXA9 mRNA ( $p=0.548$, Chi-square test). Similarly, in the 25 methylation probes encompassing the HOXA9 locus that were evaluated, most presented a consistent methylation pattern across all patients (Figure 1D). Together, these results suggest that gene amplification or methylation are not major drivers of HOXA9 overexpression in glioma.

We have recently associated high levels of HOXA9 expression with shorter overall survival (OS) of patients with GBM [16]. In order to validate this finding in a significantly larger and independent dataset, the prognostic value of $H O X A 9$ was evaluated in 554 GBM patients with available survival data from TCGA [17]. Patients whose tumors present high HOXA9 expression had significantly shorter OS (median OS 323 days; 95\% CI 183-463 days) than patients whose tumors express low HOXA9 levels (median OS 430 days; 95\% CI 396-464 days; Logrank test $p$-value $=0.017$; Figure $1 \mathrm{E}$ ). Importantly, this association between HOXA9 and OS was independent of other putative prognostic variables, including patient age, gender, Karnofsky performance status, MGMT methylation and treatment with chemotherapy, as indicated by multivariate analysis (Cox model $p$-value $=0.017$; Figure 1F). Interestingly, in GBM patients presenting low levels of MGMT expression, HOXA9 overexpression alone was sufficient to identify a subset of patients with shorter OS (median OS of $291 \pm 75.4$ and $435 \pm$ 19.2 days in HOXA9-positive versus HOXA9-negative patients, respectively; Log-rank, $p$-value $=0.013$ ). The 


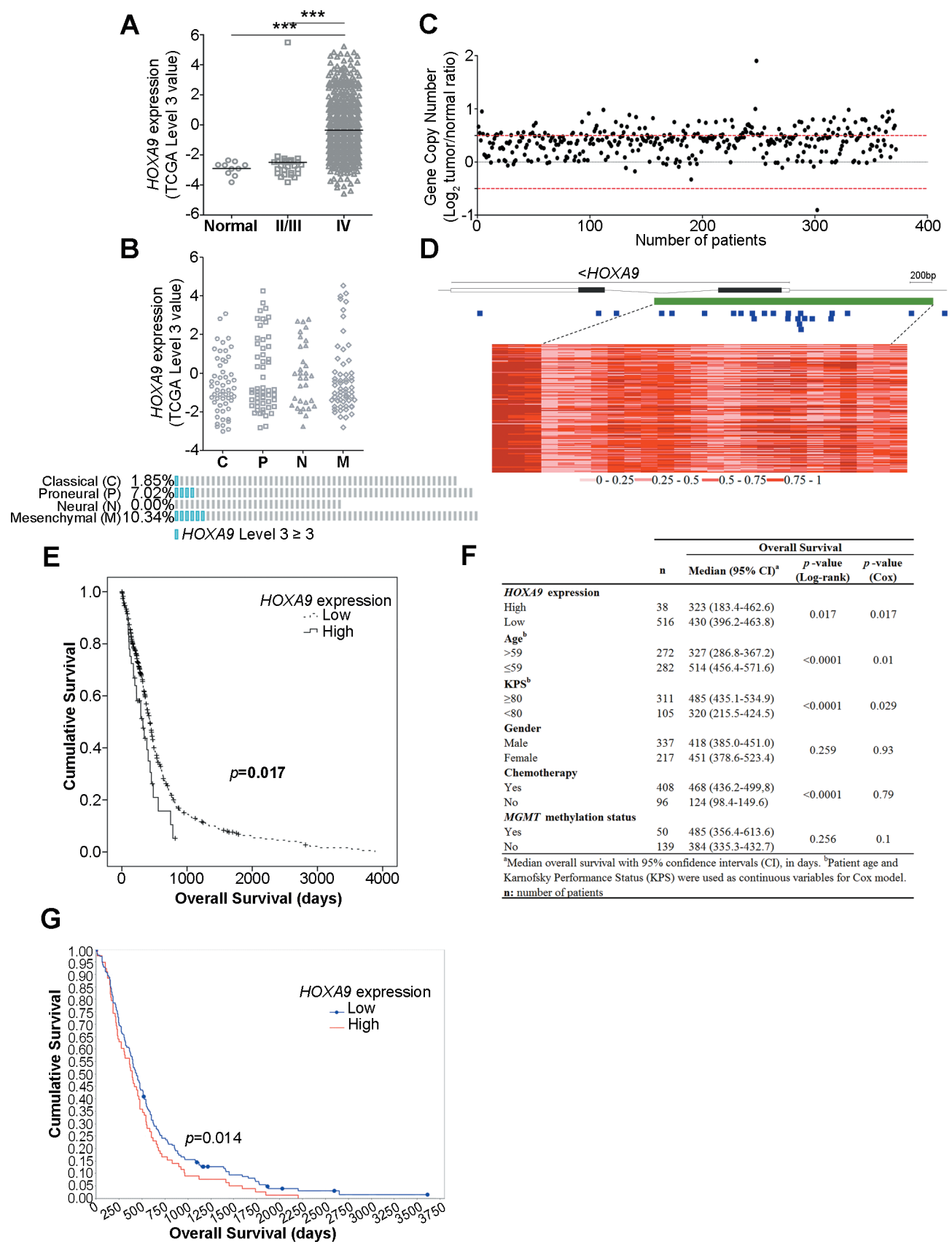

Figure 1: HOXA9 expression is associated with WHO glioma grade and is an independent prognostic factor in glioblastoma patients. (A) Expression levels of HOXA9 in 10 unmatched normal controls, 27 lower-grade gliomas (LGG) and 572 glioblastoma (GBM) patients from TCGA. HOXA9 is significantly overexpressed in GBM patients compared to LGG or normal samples $(* * *=p<0.0001)$. (B) HOXA9 high expression (TCGA level $3 \geq 3$ ) is more frequent in the mesenchymal $(10.34 \%)$ and in the proneural subtypes (7.02\%). (C) HOXA9 gene copy number status in 372 GBM specimens from TCGA. HOXA9 is amplified ( $\log _{2}$ Copy Number Tumor/Normal $\geq 0.5)$ in $31 \%(n=114)$ of GBM samples. The normal copy number interval is between the red dashed lines. (D) Heatmap representation of DNA methylation levels (TCGA $\beta$-values) of the chromosomal region encompassing HOXA9 in 74 GBM samples from TCGA. A total of 25 methylation probes (blue squares) were assessed, encompassing the $\mathrm{CpG}$ island (> $300 \mathrm{bp}$, represented in green). The color code (grades of red color corresponding to different methylation indexes) is shown below the heatmap. Each line corresponds to a patient and each column to a probe. The lines within HOXA9 correspond to introns. (E) Kaplan-Meier survival curves of 554 GBM patients from TCGA indicate that patients whose tumors present high levels of HOXA9 expression show a statistically significant shorter overall survival when compared to those whose tumors present lower levels of $H O X A 9$ (Log-rank test, $p$-value $=0.017$ ). (F) Univariate and multivariate analyses of associations between HOXA9 expression levels and survival of patients with glioblastoma (adjusted for patient age, KPS, gender, treatment with chemotherapy, and MGMT methylation status). (G) Kaplan-Meier survival curves of 181 GBM patients from REMBRANDT dataset confirms that patients whose tumors present high levels of HOXA9 expression (78/181) present a statistically significant shorter overall survival when compared to those whose tumors present lower levels (Log-rank test, $p$-value $=0.014$ ). 
prognostic value of $H O X A 9$ was additionally validated in an independent dataset from the REMBRANDT database (Log-rank, $p$-value $=0.014$; Figure $1 \mathrm{G})$.

Together, these data implicate HOXA9 as a critical molecule in the malignant progression of gliomas, and validate its prognostic value in high-grade GBM patients.

\section{HOXA9-mediated transcriptomic signatures in GBM sustain cancer-related pathways}

The endogenous expression levels of HOXA9 in a panel of glioma cell lines was evaluated by qPCR (Supplementary Figure 1). In order to provide the first characterization of HOXA9 targets on a genome-wide level in GBM, expression microarray analyses were performed in matched HOXA9-positive and HOXA9negative human GBM cell models (U87MG-MSCV vs. U87MG-HOXA9, human immortalized astrocytes hTERT/E6/E7-MSCV vs. hTERT/E6/E7-HOXA9, U251shControl vs. U251-shHOXA9, and a primary GBM cell line GBML18-shControl vs. GBML18-shHOXA9; Figure 2A and 2B). Due to HOXA9 expression, 3454 probes were significantly differentially expressed in U87MG cells (1537 upregulated and 1917 downregulated), 417 probes in hTERT/E6/E7 cells (166 upregulated and 251 downregulated), 2452 probes in U251 cells (1301 upregulated and 1151 downregulated), and 5886 probes in GBML18 patient-derived primary cells (2802 upregulated and 3084 downregulated; Figure 2B and Supplementary Figures 4-9; GEO accession number GSE56517). Only a small subset of probes was consistently altered in the 3 GBM cell lines (17 probes upregulated and 40 downregulated due to HOXA9 expression in U87MG, U251 and GBML18 cells), which, as expected, was even smaller when integrating the non-tumor immortalized astrocytes (1 probe upregulated and 3 downregulated; Figure 2B), indicating that the transcriptome of HOXA9 is cell-type dependent, as previously suggested in leukemia models [19]. Gene-specific expression analyses in a subset of HOXA9 targets validated the array data (Supplementary Figure 2A-2D).

In order to understand the biological relevance of the HOXA9 transcriptome, functional clustering annotation and integration into Kyoto Encyclopedia of Genes and Genomes (KEGG), Reactome and Gene Ontology (GO) analyses were performed (Figure 2C-2F and Supplementary Figure 3A-3G). The HOXA9 transcriptome of non-tumoral hTERT/E6/E7 immortalized astrocytes revealed particular enriched pathways in KEGG (e.g. chemokine signaling pathway for HOXA9-upregulated genes, and cytokine-cytokine receptor interaction pathway for HOXA9-downregulated genes; Supplementary Figure 3A). These pathways have been found deregulated in the context of cancer, and associated with increased proliferation and invasion [20]. In the GO integration (Figure 2C), these cells showed significant enrichment mainly to biological processes related to cellular adhesion (HOXA9-upregulated genes) and regulation of protein activity (HOXA9-downregulated genes). No significant enrichments were observed for the Reactome pathways in these cells. In the 3 GBM cell lines, the HOXA9 transcriptomes were enriched for several cancer-related pathways in KEGG, GO and Reactome analyses, including genes involved in cell cycle, DNA replication and repair, RNA processing, cell adhesion and migration, vasculature development, and immune-related pathways (Figure 2D-2F and Supplementary Figure 3B$3 \mathrm{G})$. Together, these data suggest the HOXA9-mediated genetic signatures in GBM cell models are associated with important cancer hallmarks that may favor tumor development, progression, and aggressiveness. Moreover, HOXA9-target genes may be important in sustaining the HOXA9-associated aggressive phenotype and poor prognosis observed in GBM patients.

We next explored the Connectivity Map tool to identify drugs that induce gene expression signatures similar to our HOXA9 transcriptome data. Interestingly, the top 30 gene expression signatures revealed associations with cancer cells treated with phosphatidylinositol 3-kinase (PI3K) pathway inhibitors, namely LY-294002, sirolimus, and wortmannin (Supplementary Figure $3 \mathrm{H}$ ), suggesting that our HOXA9 transcriptomic signature in GBM cell models is modulated by these drugs. This is in accordance with our previous report showing that inhibition of the PI3K pathway in GBM cells inhibits HOXA9 transcription [16], further supporting the validity of our microarray data. Other drug treatments that alter HOXA9-associated gene expression signature were found among the tested cell lines (Supplementary Figure $3 \mathrm{H}$ ), including several anti-cancer drugs, particularly histone deacetylase (HDAC) inhibitors trichostatin A (TSA), vorinostat and MS-275 [21], which are able to promote cell cycle arrest and apoptosis [22], and tanespimycin and its analogues geldanamycin, alvespimycin, and monorden $[23,24]$, which are HSP90 inhibitors also currently tested as anti-cancer drugs. Together, our findings indicate that the HOXA9 transcriptome in GBM cells may be reverted by several drugs with potential anticancer effects, particularly those interfering with PI3K signaling, HDAC and HSP90 functions, which may be clinically valuable to revert HOXA9-driven transcriptomic signatures.

\section{HOXA9 expression is associated with cancer stem cell features}

HOXA9-associated transcriptional signatures queried with gene set enrichment analysis (GSEA) revealed that the HOXA9 transcriptome in hTERT/E6/E7 cells was inversely associated with genes downregulated in glioma stem cells (enrichment score, $\mathrm{ES}=-0.54$, false discovery rate, FDR $=0.19$; Figure $3 \mathrm{~A}$ ), leukemia stemlike cells, and hematopoietic stem cells $(\mathrm{ES}=-0.44$, 

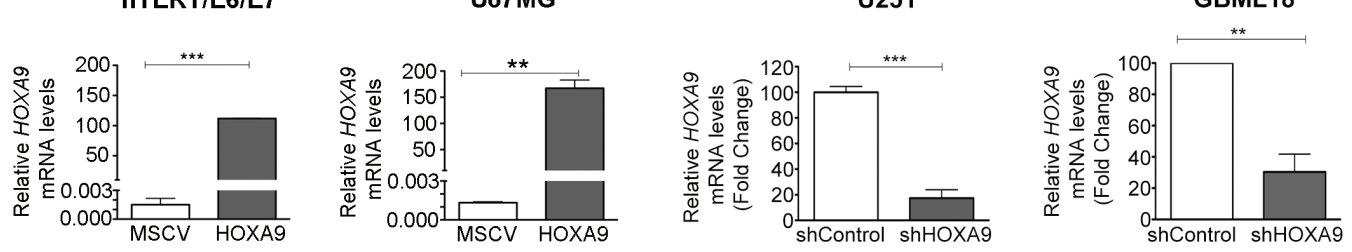

B

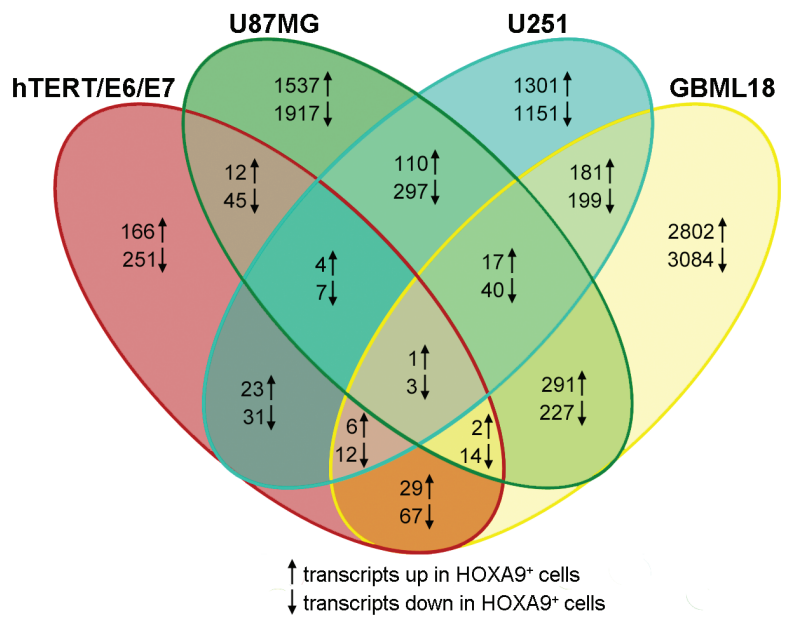

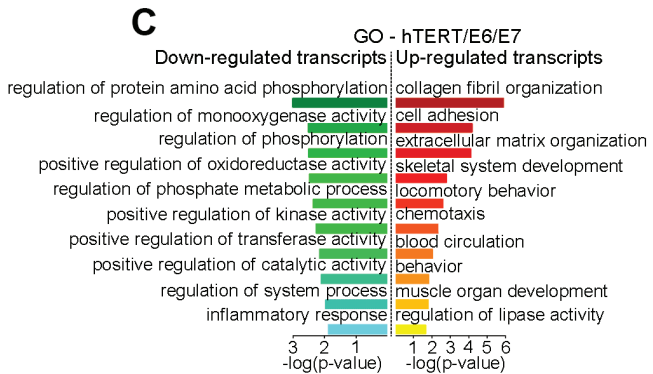

$E$ Down-regulated transcripts: Up-regulated transcripts

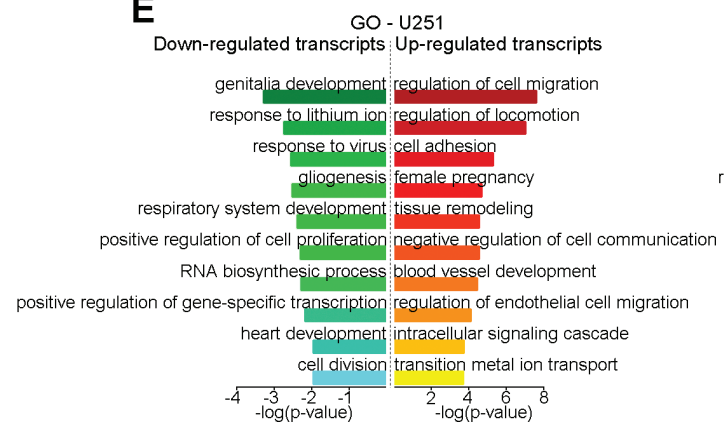

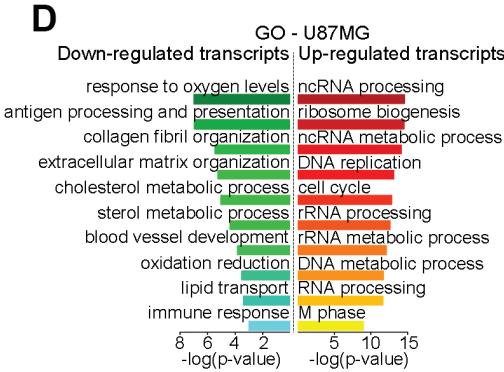

F GO - GBML18
Down-regulated transcripts
ncR-regulated transcripts
ribosome biogenesis
bone development
bell adhelllular signaling cascade regulation of megakaryocyte deffierentiation $:$ Ras protein signal transduction forebrain neuron fate commitment heart development translation response to metal ion

macromolecular complex assembly urogenital system development transcription response to inorganic substance rRNA metabolic process vasculature development activation of protein kinase activity phosphate metabolic process $-4 \underset{-\log (p-v a l u e)}{-3-1}-\frac{2}{2 \underset{-\log (p-v a l u e)}{6}} 8$

Figure 2: HOXA9 transcriptomes in hTERT/E6/E7 human immortalized astrocytes, and in U87MG, U251 and GBML18 glioblastoma cell lines. (A) qPCR confirming the overexpression of HOXA9 in hTERT/E6/E7-HOXA9 and U87MGHOXA9 cells and HOXA9-silencing in U251-shHOXA9 and GBML18-shHOXA9 cells, comparing to their respective control counterparts. (B) Venn diagram summarizing the number of differentially expressed transcripts in the microarray data in all cell lines. The numbers in each area represent the total number of transcripts within each intersection. (C-F) DAVID was used to query the HOXA9-transcriptome from each cell line (C, hTERT/E6/E7; D, U87MG; E, U251; F, GBML18), in order to identify enriched biological terms on the differentially expressed genes extracted from the microarray data. Statistically significant enriched GO terms are shown for each cell line. 
A

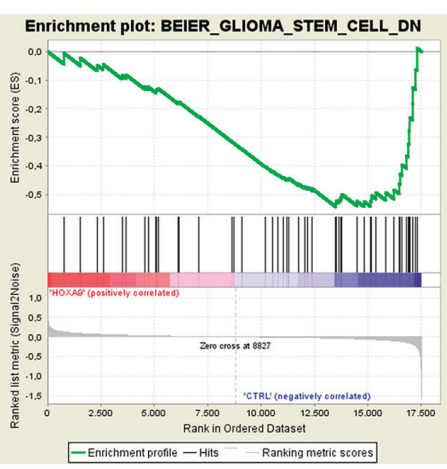

C

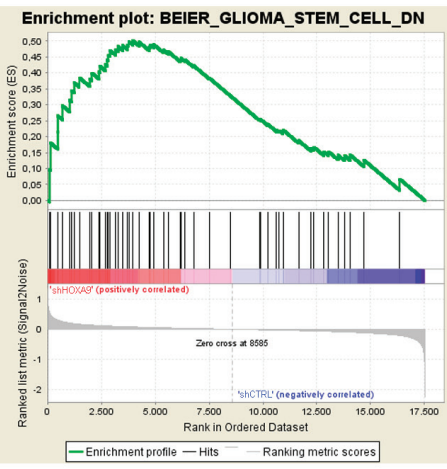

B

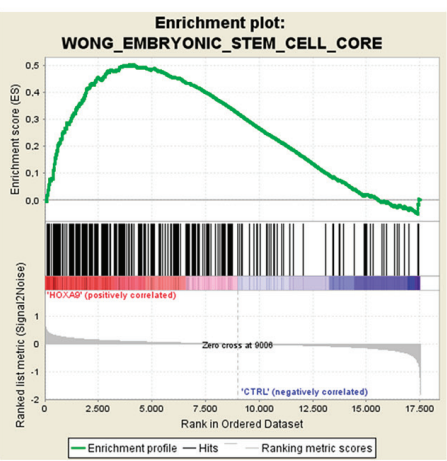

D

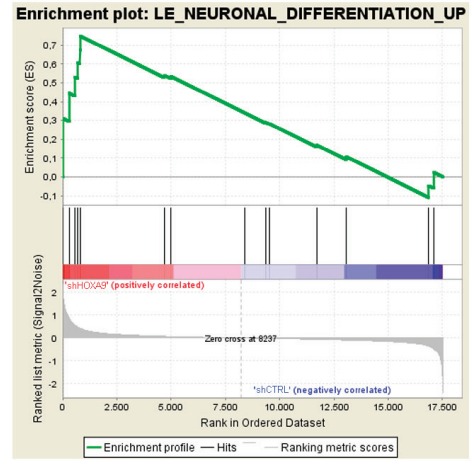

E

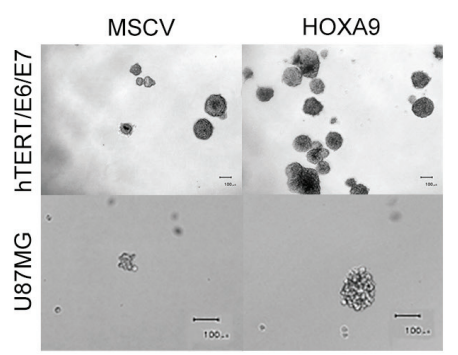

$\mathbf{F}$

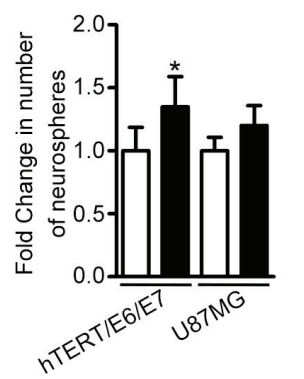

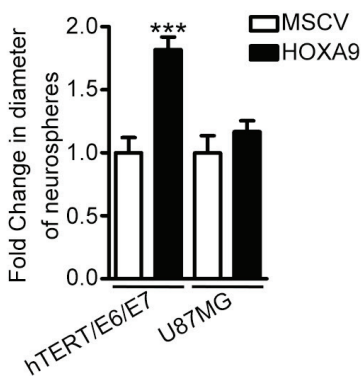

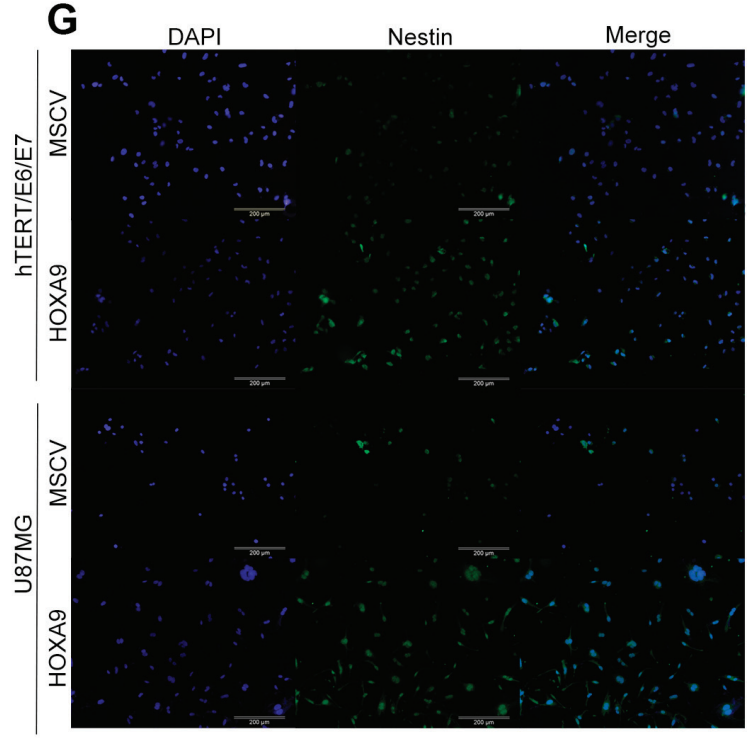

Figure 3: HOXA9 transcriptomes are associated with cancer stem cell features. (A-D) GSEA reveals that the HOXA9 transcriptomes in hTERT/E6/E7 (A) and U251 (C) cells are associated with transcriptional signatures of glioma stem-like cells (Enrichment Score, ES $=-0.54$, False Discovery Rate, FDR $=0.19$; and ES $=0.50, \mathrm{FDR}=0.11$, respectively); in U87MG cells $(\mathbf{B})$, the HOXA9 transcriptome is positively associated with genes that are upregulated in embryonic stem cells ( $\mathrm{ES}=0.50$, FDR $<0.0001$ ); in GBML18 cells (D), HOXA9 transcriptome is inversely associated with genes upregulated during the neuronal differentiation $(\mathrm{ES}=0.75, \mathrm{FDR}=0.21)$. (E) Representative phase contrast photographs of hTERT/E6/E7 and U87MG neurospheres are shown. (F) Quantification of neurospheres number and size for each cell line $\left(n=3 ;{ }^{*} p<0.05 ; * * * p<0.001\right)$. (G) Immunofluorescence showing increased Nestin staining in HOXA9positive cells. 
$\mathrm{FDR}=0.21 ; \mathrm{ES}=-0.45, \mathrm{FDR}=0.20$, respectively; Supplementary Figure 10A and Supplementary Table 1). Concordantly, HOXA9 target genes in U87MG cells were significantly associated with genes upregulated in embryonic stem cells $(\mathrm{ES}=0.50, \mathrm{FDR}<0.0001$; Figure 3B), DNA repair, and cycling gene expression signatures $(\mathrm{ES}=0.54, \mathrm{FDR}<0.0001$; and $\mathrm{ES}=0.38$, FDR $=0.007$, respectively; Supplementary Figure 10B and Supplementary Table 1). Similarly, the transcriptome of HOXA9-silenced U251 cells is significantly associated with genes depleted in glioma stem cells and normal (embryonic, neural and hematopoietic) stem cells $(\mathrm{ES}=0.50, \mathrm{FDR}=0.11$ and $\mathrm{ES}=0.43, \mathrm{FDR}=0.22$, respectively; Figure 3C, Supplementary Figure 10C and Supplementary Table 1); in GBML18 cell line, HOXA9downregulation enriched for genes upregulated during neuronal differentiation and in quiescent chronic myeloid leukemia cells $(\mathrm{ES}=0.75, \mathrm{FDR}=0.21$ and $\mathrm{ES}=0.70$, FDR $=0.21$, respectively; Figure $3 \mathrm{D}$, Supplementary Figure 10D and Supplementary Table 1). Together, these analyses suggest that the HOXA9-transcriptome is enriched for genes involved in stem-like cell features, a critical hallmark of cancer. To test this hypothesis, we evaluated the capacity of these cells to form neurospheres in vitro, which has been associated with stemness potential [25]. HOXA9 significantly increased the size and number of neurospheres in hTERT/E6/E7 cells as compared to their HOXA9-negative counterparts, which was also observed in a lower extent in U87MG cells (Figure 3E and 3F). Additionally, the expression of the neural stem cell marker Nestin was significantly higher in both hTERT/E6/E7 and U87MG HOXA9 positive cells (Figure $3 \mathrm{G})$. Together, these data suggest HOXA9 increases the stemness capacity of these cells.

\section{$H O X A 9$ expression is associated with tumor aggressiveness in GBM xenograft models}

Considering that stem cell properties have been linked with tumor malignancy $[14,26,27]$, together with our microarray data implicating the HOXA9 transcriptome in typical hallmarks of cancer (Supplementary Figure 3, Supplementary Figure 10 and Supplementary Table 1), we hypothesized that HOXA9 could play a role in tumor growth in vivo. To test this hypothesis, we established subcutaneous GBM xenograft models with U87MG cells. Tumors derived from U87MG-HOXA9 cells presented increased growth rates and were significantly larger than U87MG-MSCV cells (Figure 4A-4E). Interestingly, the final tumor volume was significantly correlated with $H O X A 9$ expression levels (Figure 4C), further strengthening the relevance of $H O X A 9$ on tumor growth kinetics in vivo. Histologically, $H O X A 9$ positive tumors presented a higher proliferative activity, as indicated by increased Ki-67 staining, which includes mostly neoplastic cells but also non-neoplastic cells (e.g. endothelial and stromal cells; Figure 4F). A higher expression of Nestin was also observed, supporting an increased stemness potential of HOXA9-positive cells in vivo. Furthermore, the expression levels of Cyclin D1 and BCL2 were also increased in U87MG-HOXA9 tumors in vivo (Figure 4F). These proteins are critical regulators of cell cycle and cell death, respectively, and were identified as HOXA9 targets in the microarray data. Tumor-associated angiogenesis, another critical hallmark of GBM, as assessed by PECAM1 protein staining, was significantly increased in HOXA9-positive tumors (Figure 4F and 4G). Together, these data establish $H O X A 9$ as a critical mediator of GBM growth and aggressiveness in vivo, and identifies critical molecular mediators sustaining this malignant phenotype.

\section{$H O X A 9$ expression contributes to glioma initiation and causes glioma-associated death}

Given our data implicating HOXA9 as a key molecule in mediating several aspects of cancer aggressiveness and stem cell characteristics, we hypothesized that HOXA9 may also play an important role in the tumorigenic process, possibly facilitating carcinogenesis initiation. To test this hypothesis, we evaluated the influence of HOXA9 on the capacity of hTERT/E6/E7 cells, a non-tumorigenic cell line [28], to establish tumors in vivo. In a subcutaneous model, hTERT/E6/E7 cells did not form tumors, regardless of HOXA9 expression status (Figure 5A). Considering the relevance of the interplay between tumor cells and its microenvironment, we investigated the tumorigenic potential of these cells in an orthotopic intracranial model. Strikingly, while HOXA9-negative hTERT/E6/E7 cells did not form tumors, the expression of HOXA9 in hTERT/E6/ E7 rendered these cells highly tumorigenic in the brain, accompanied by glioma-related symptomatology and death (Figure 5A-5E). Histological analyses of the brains revealed that hTERT/E6/E7-HOXA9 tumors displayed hallmark features of malignant gliomas, including pleomorphic and spindle shape tumor cells, with prominent nuclear polymorphism and mitotic activity, and were highly infiltrative throughout the meninges and brain parenchyma. In some cases, tumors grew outside the brain, infiltrating bone and soft tissues, where cells displayed a more sarcomatoid appearance (Figure 5D). These data clearly establish HOXA9 as a novel critical molecular driver in the initial steps of malignant transformation of brain gliomas.

\section{$H O X A 9$ increases GBM aggressiveness by affecting cell viability, death, invasion and resistance to temozolomide in vitro}

Our data implicated HOXA9 in several hallmarks of cancer, including cell proliferation, invasion, DNA repair pathways, and cancer stem cell features (Figure 3, Supplementary Figure 3 and Supplementary Figure 10 and Supplementary Table 1). Since most of 
A

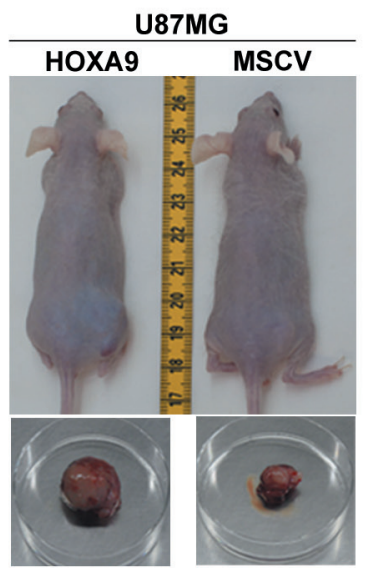

B

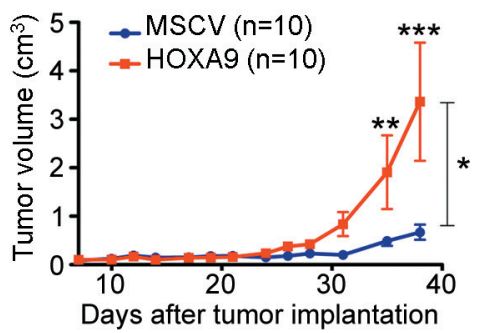

C

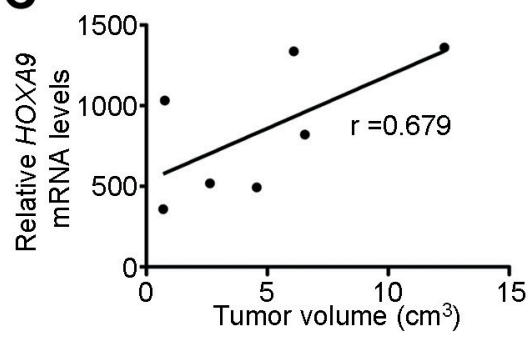

D

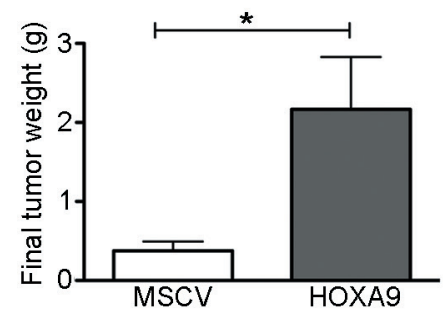

E

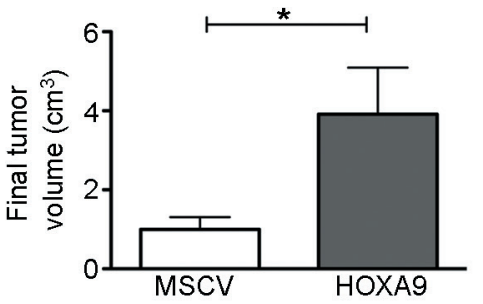

F

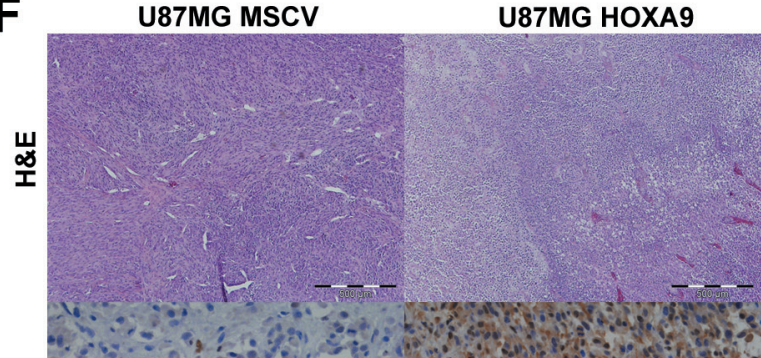

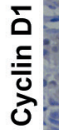

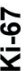

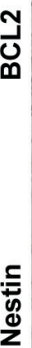

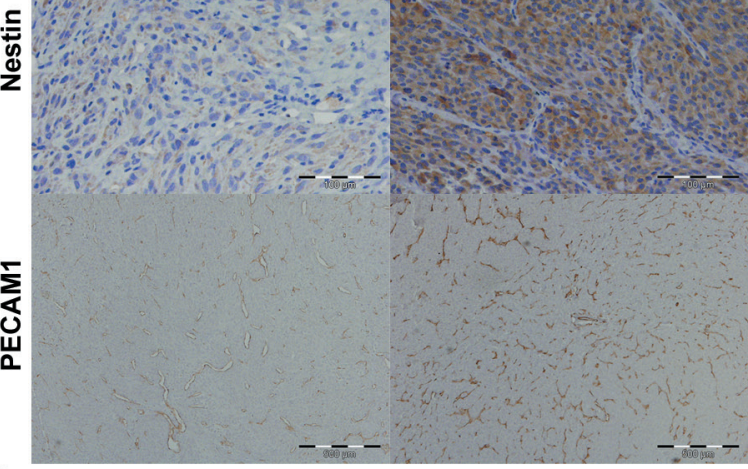

G

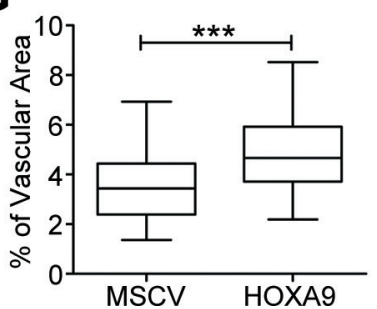

Figure 4: HOXA9 accelerates tumor growth in vivo. (A) U87MG cells were subcutaneously xenografted into nude mice; representative photographs of in vivo and ex vivo tumors are shown. (B) Longitudinal assessment of tumor volumes showing increased growth rates of U87MG-HOXA9 tumors as compared to HOXA9-negative tumors. (C) Positive correlation between expression levels of HOXA9 and tumor volume. (D) and (E) Final tumor volumes (D) and weights (E) are significantly higher in HOXA9-positive tumors $(n=10)$. (F) Hematoxilin-eosin and immunohistochemical staining showing that HOXA9-positive tumors present higher protein levels of Cyclin D1, Ki-67, BCL2, Nestin, and PECAM1. (G) Quantification of the \% of vascular area based on PECAM1 staining, showing increased angiogenic potential of U87MG-HOXA9 tumors. Statistical differences were calculated by two-way ANOVA (B) and Spearman correlation (C), or $t$-tests (D and E) $(* p<0.05 ; * *<0.01 ; * * p<0.001)$. 

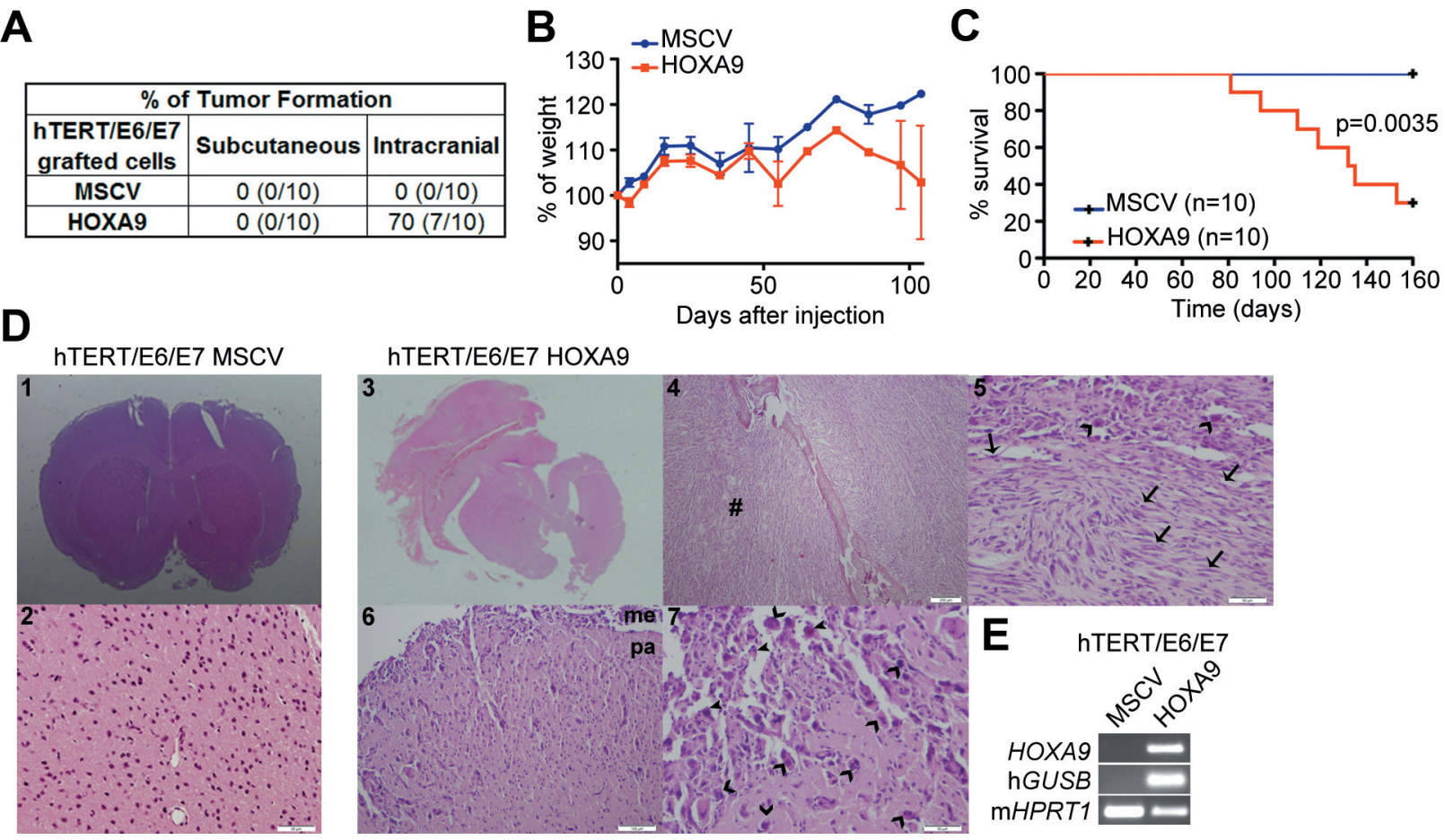

Figure 5: $H O X A 9$ induces glioma initiation and tumor-associated death in intracranial orthotopic xenografts. (A) Nude mice were injected with $H O X A 9$-negative and HOXA9-positive hTERT/E6/E7 immortalized astrocytes, either subcutaneously or intracranially. No tumor formation was observed in the subcutaneous model, regardless of HOXA9 expression. In the intracranial orthotopic model, HOXA9-positive hTERT/E6/E7 cells originated tumors in the majority of tested mice (70\%). (B) Mice intracranially injected with hTERT/E6/E7-HOXA9 cells display glioma-related loss of weight. (C) Kaplan-Meier survival curves showing tumor-associated death exclusively in mice bearing HOXA9-positive cells (Log-rank test, $p=0.0035$ ). (D) Histological characterization of mice brains orthotopically-injected with hTERT/E6/E7-control or hTERT/E6/E7-HOXA9 cells. Brains from animals injected with HOXA9-negative cells display a normal, non-malignant appearance (images 1 and 2 at 20x and 200x magnification, respectively), while HOXA9-positive cells formed tumors displaying characteristic hallmarks of malignant gliomas (images 3, 4, 5, 6 and 7 at the magnification of 20x, 40x, 200x, 100x and 200x, respectively), including pleomorphic and spindle shape tumor cells (arrows), high mitotic activity (open arrowheads), prominent nuclear polymorphism (closed arrowheads), and infiltration to meninges and brain parenchyma (6 and 7). In image 4, cells infiltrating bone and soft tissues (\#) presented a more sarcomatoid appearance, amplified in image 5. (E) RT-PCR analysis confirmed the expression of human GUSB housekeeping gene and HOXA9 exclusively in HOXA9-positive xenografts. $\mathrm{Me}=$ meninges; pa = brain parenchyma.

these characteristics have been implicated in resistance to treatments, we performed an integrated set of functional assays to investigate HOXA9's effects on these processes, both in basal conditions and under temozolomidetreatment, the gold standard chemotherapeutic agent for GBM patients. Both hTERT/E6/E7 and U87MG cells overexpressing HOXA9 presented significantly higher temozolomide $\mathrm{IC}_{50}$ values than their respective control cell lines (Figure 6A). In agreement with the overexpression models, we observed a decrease in temozolomide $\mathrm{IC}_{50}$ values for both cell lines after HOXA9 downregulation (Figure 6B). Time-course viability assays were further evaluated by trypan blue exclusion (Figure 6C-6F) and MTS reduction (Supplementary Figure 11A-11D). Globally, both assays revealed that all HOXA9-high cell lines hTERT/E6/E7, U87MG, U251, and GBML18 (red lines) presented higher cell viability in basal conditions than their respective HOXA9-low counterparts (blue lines; Figure $6 \mathrm{C}-6 \mathrm{~F}$ and Supplementary Figure 11A-11D), and that temozolomide-mediated cytotoxicity was significantly more pronounced in HOXA9-low cells than in $H O X A 9$-high cells (Figure 6C-6F and Supplementary Figure 11A-11D), collectively indicating that HOXA9 contributes to temozolomide resistance in GBM cells. We next assessed the influence of HOXA9 expression in cell death by annexin $\mathrm{V}$ staining followed by flow cytometry. In basal conditions, most HOXA9-high cells presented significantly lower levels of cell death than their respective HOXA9-low counterparts (Figure 6G). In addition, all HOXA9-high cells presented significantly lower levels of temozolomide-mediated cell death than HOXA9-low cells (Figure 6G). An important feature of GBM is its striking ability to invade adjacent brain tissue, which is a major cause of tumor recurrence and resistance to therapy. Using invasion assays, we observed that HOXA9 significantly increases the invasive capacity of hTERT/E6/E7, U87MG, and GBML18 cells, both in basal conditions and after treatment with temozolomide. In 

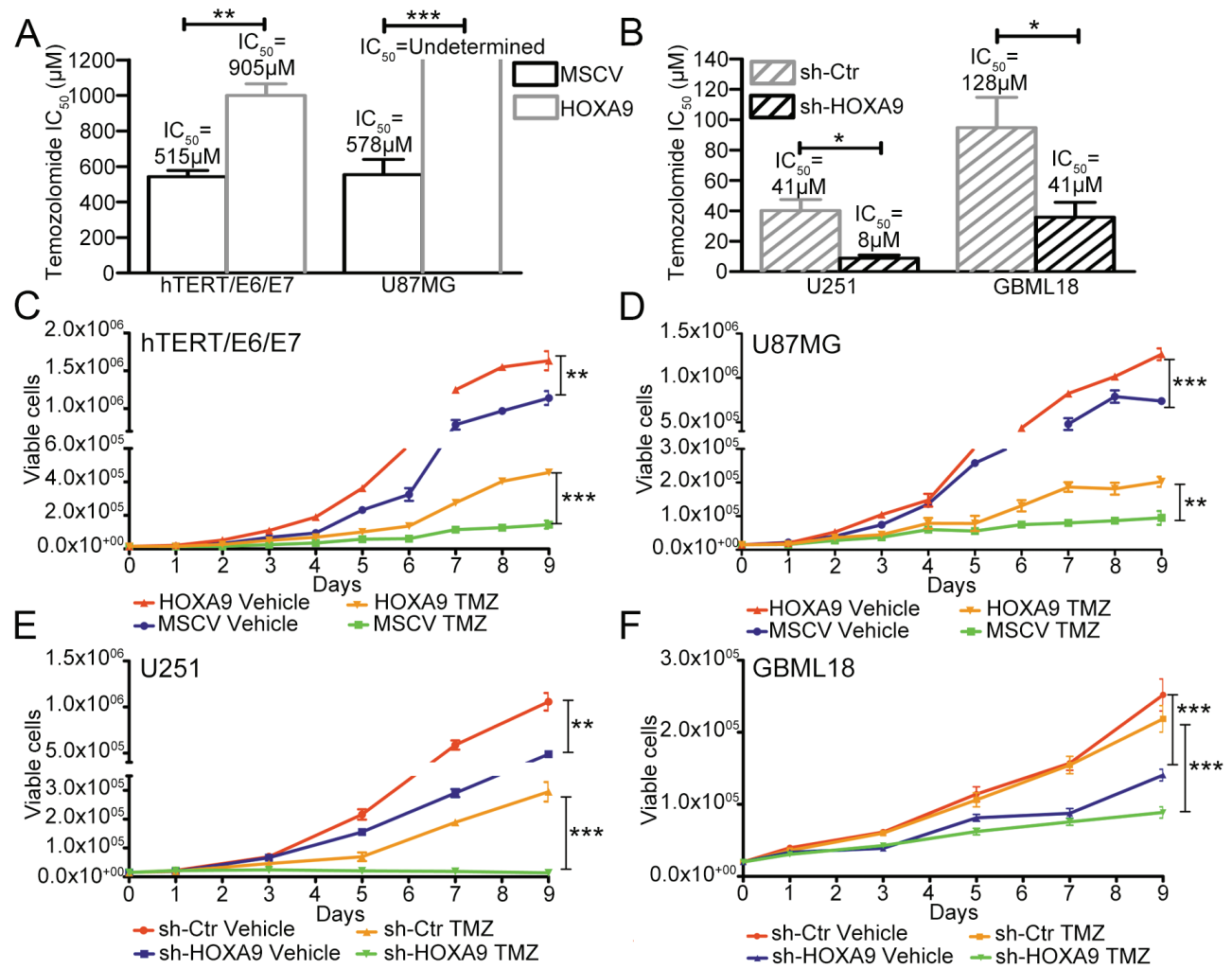

G
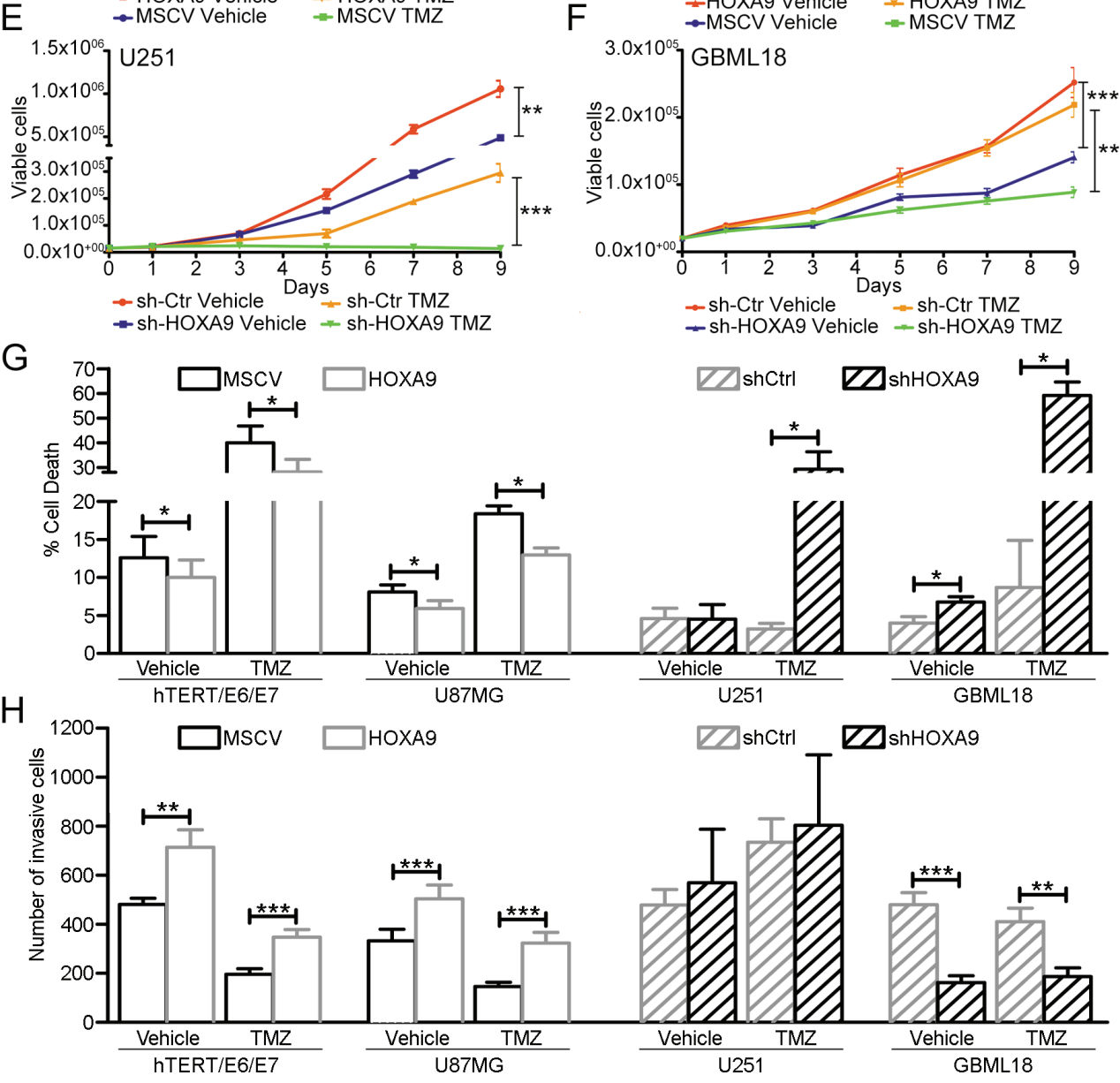

Figure 6: Functional roles of $H O X A 9$ in GBM cell viability, death, and invasion, under basal conditions and temozolomide treatment. (A and B) Determination of the half inhibitory concentration $\left(\mathrm{IC}_{50}\right)$ values after 6 days of temozolomide (TMZ) treatment in HOXA9-positive or HOXA9-negative hTERT/E6/E7 and U87MG (A), and HOXA9-silenced or control U251 and GBML18 (B) cell lines. (C-F) Cell viability trypan blue assays in HOXA9-negative/low or HOXA9-positive/high hTERT/E6/E7 (C), U87MG (D), U251 (E) and GBML18 (F) cells, exposed to temozolomide or vehicle. (G) Cell death was evaluated by annexin V staining followed by flow cytometry in HOXA9-positive/high and HOXA9-negative/low hTERT/E6/E7, U87MG, U251 and GBML18 cell lines, both in basal conditions and after exposure to temozolomide (TMZ). HOXA9 expression decreases cell death of all GBM cell models, both in basal conditions and after TMZ treatment, except in basal conditions for U251 cell line. (H) Cell invasion in the same cell lines, both in basal conditions and after exposure to TMZ. HOXA9 increases the invasion of hTERT/E6/E7, U87MG, and GBML18 cells. U251 cells did not show significant differences in invasion profiles due to HOXA9 levels or TMZ treatment. Results are representative of at least three independent experiments, performed in triplicates (data points represent mean \pm SEM). Statistical differences were calculated by Student's $t$-test (panels A, B, G, H) and two-way ANOVA (panels C-F) $\left({ }^{*} p<0.05 ; * * p<0.01 ; * * *<0.001\right.$ ). 
contrast, no significant changes in invasion were observed in U251 cells (Figure 6H). Since invasion and migration are intimately-related cellular processes, we also evaluated the migration capacity of U251 cells by wound healing assays (Supplementary Figure 12). Interestingly, U251shControl cells presented significantly higher migration rates than U251-shHOXA9 counterparts. Together, our data consistently establishes the functional relevance of HOXA9 expression in several hallmarks of GBM cells behavior, by affecting key cellular processes such as cell viability, death, invasion, and drug resistance, which together may dictate the more aggressive behavior and poorer clinical outcome of HOXA9-positive GBMs.

\section{$H O X A 9$ expression associates with shorter survival and increased resistance to temozolomide in orthotopic GBM xenograft models}

In order to validate the prognostic value of HOXA9 observed in GBM patients (Figure $1 \mathrm{E}-1 \mathrm{G}$, and [16]), and its relevance in temozolomide chemo-resistance observed in vitro (Figure 6 and Supplementary Figure 11), we established intracranial orthotopic GBM xenografts with U87MG-MSCV and U87MG-HOXA9 cells in nude mice (Figure 7A). Untreated animals bearing U87MGHOXA9-derived tumors presented significantly lower overall survival (median 28 days) than their respective negative counterparts (median 46 days; Log rank test, $p=0.0001$; Figure 7A). Additionally, while treatment with temozolomide was able to significantly extend overall survival of all animals (median OS increased from 46 to 140 days in mice bearing U87MG-MSCV tumors, $p<0.0001$; and median OS from 28 to 65 days in mice bearing U87MG-HOXA9 tumors, $p=0.0002$; Figure 7A), those bearing $H O X A 9$-expressing tumors presented a significantly shorter overall survival than animals bearing HOXA9-negative tumors ( $p<0.0001$; Figure 7A). Histological and RT-PCR analyses confirmed tumor formation and HOXA9 expression levels in tumors excised from mice brains (Figure 7B and 7C, respectively). Taken together, our in vivo experiments specifically establish HOXA9 as a solid biomarker of prognosis in GBM and its relevance in determining temozolomide chemo-resistance.

\section{HOXA9 affects important pathways involved in temozolomide response}

The most well established mechanisms of temozolomide resistance include MGMT, base-excision repair (BER), and mismatch-repair (MMR) proteins [29]. In order to understand the mechanisms by which HOXA9 may affect drug resistance and mice survival, we investigated if HOXA9 affects these key pathways. By using methylation-specific PCR, we observed that U87MG cells presented a methylated $M G M T$ promoter, independently of HOXA9 expression (Supplementary Figure 13A). Concordantly, no differences in $M G M T$ mRNA expression and protein levels were detected (Supplementary Figure 13B and 13C). Thus, the HOXA9mediated temozolomide resistance in U87MG cells is not likely mediated by MGMT. Similarly, the levels of BER proteins (PCNA, PARP1, APEX1, and XRCC1) were not significantly affected by $H O X A 9$ (Supplementary Figure 13D). In contrast, the expression of some key proteins of the MMR pathway, including PMS2 and MSH6, was significantly decreased due to HOXA9 (Figure 7D). Since a functional MMR pathway is crucial for temozolomide sensitivity by inducing apoptosis after DNA damage, the down-regulation of these key MMR proteins in HOXA9positive U87MG cells may be an additional mechanism of temozolomide resistance.

Critically, it is not straightforward to therapeutically intervene at proteins of the MMR pathway in the clinical setting. Thus, we revisited our microarray data to identify other putative mechanisms of drug resistance. Interestingly, we identified several genes involved in apoptosis and drug response that were differentially expressed in U87MGHOXA9 cells (e.g., upregulation of anti-apoptotic BCL2, TRAIP, BAG1, BAG5, API5, SET; and downregulation of pro-apoptotic $N L R P 1, B L I D, A P L P 1, A D C K 3)$. Of these, $B C L 2$ was particularly interesting because it was one of the top 50 most significantly up-regulated genes in our HOXA9 array data (Supplementary Figure 5). BCL2 is a critical anti-apoptotic protein involved in resistance to DNA damaging agents in many cancer types [30], for which pharmacological inhibitors are available, some of which have shown promising results in the treatment of solid tumors [31, 32]. Thus, we investigated how HOXA9 may affect temozolomide response via BCL2. We first confirmed that BCL2 is significantly overexpressed at the mRNA and protein levels in HOXA9-positive cells (Figure 7E and $7 \mathrm{~F}$ ), which was consistent with increased levels of BCL2 protein in HOXA9-expressing subcutaneous tumor (Figure 4F). In order to confirm the link between HOXA9-mediated BCL2 overexpression and resistance to temozolomide, and to explore novel combinatorial treatments to GBM, we evaluated how ABT-737, a potent small molecular inhibitor of BCL2 family proteins, affects the response of U87MG cells to temozolomide. While no significant cytotoxic effects were observed for two different doses of ABT-737 alone $(0.25$ and $5 \mu \mathrm{M})$, the combination of ABT-737 with temozolomide significantly increased cytotoxicity of U87MG cells, an effect that was significantly more pronounced in $H O X A 9$-positive cells (Figure 7G). This finding supports the evidence that BCL2 could be associated with temozolomide resistance in U87MG HOXA9-positive cells. Importantly, the use of this BCL2 inhibitor together with temozolomide did not affect cytotoxicity of cells lacking BCL2 expression (Supplementary Figure 14A and 14B), which supports the specificity of the observed effect. Importantly, in the 
A

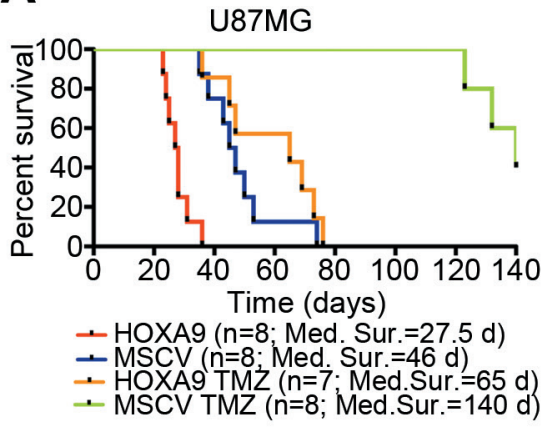

B

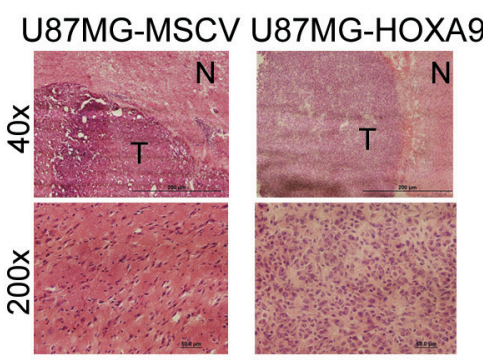

C

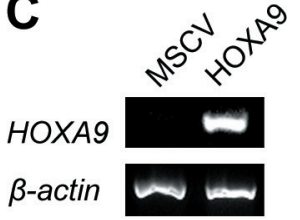

D

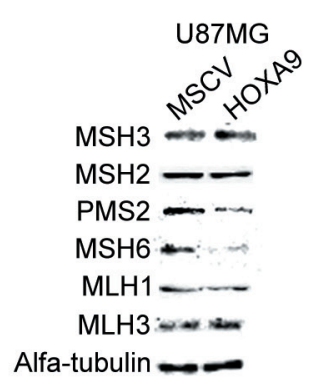

$\mathbf{E}$

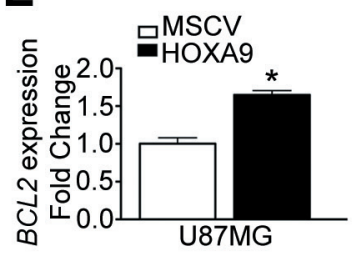

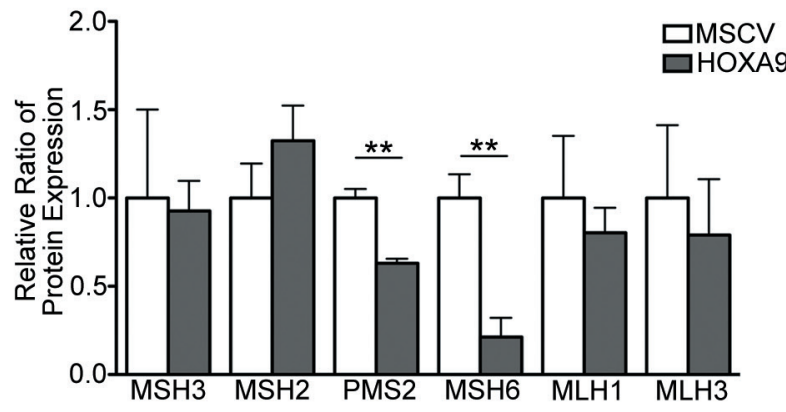

$\mathbf{F}$

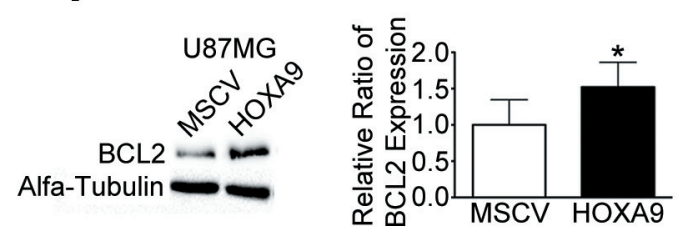

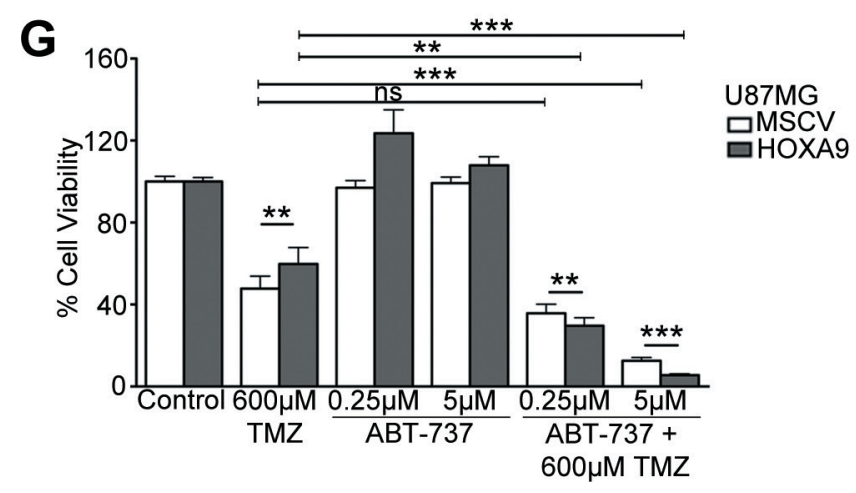

Figure 7: $\mathrm{HOX} A 9$ decreases overall survival and increases resistance to temozolomide in vivo via mismatch-repair and BCL2 proteins. (A) Kaplan-Meier survival curves for in vivo orthotopic intracranial GBM models. While temozolomide (TMZ) treatment successfully increased overall survival, HOXA9-positive tumors cause significantly decreased overall survival in both untreated and TMZ-treated animals (Log-rank test HOXA9 vs MSCV, $p=0.0001$; HOXA9 TMZ vs MSCV TMZ, $p<0.0001$ ). (B) Hematoxylineosin staining of mice brains showing a well-delimited tumor area $(T)$ with GBM-like features, and surrounding non-tumor brain tissue $(N)$. (C) RT-PCR analysis confirmed the expression of HOXA9 in brain tumors derived from U87MG-HOXA9-xenografted cells. (D) Western Blot to mismatch-repair proteins (MMR), showing that HOXA9 decreases the expression of PMS2 and MSH6 proteins in U87MG cells in vitro. (E) and (F) qPCR and Western blot analyses of BCL2 mRNA and protein levels, respectively, showing HOXA9 significantly increases BCL2 levels. Results are representative of three independent experiments for each blot. (G) Cell viability of U87MG HOXA9-negative or -positive cells after 4 days of treatment with vehicle, Temozolomide (TMZ), ABT-737 (BCL2 inhibitor), or both. HOXA9-positive cells are significantly more sensitive to the combination of TMZ and ABT-737 than HOXA9-negative cells. Results are representative of three independent experiments, performed in triplicates (data points represent mean \pm standard deviation). Statistical differences calculated by Student $t$-tests $(* p<0.05 ; * * p<0.01 ; * * *<0.001)$. 
TCGA dataset, $87 \%$ of the $H O X A 9$-high GBM patients also display high levels of $B C L 2$, emphasizing that the link between HOXA9 and BCL2 is not only present in in vitro and in vivo models of GBM, but is also relevant in the clinical setting. These data supports the future exploitation of BCL2 inhibitors combined with temozolomide for the treatment of GBM patients.

\section{DISCUSSION}

Previous studies have shown that $H O X$ genes are part of large gene expression signatures that associate with glioblastoma self-renewal and therapy response [12-14]. However, the specific contribution of individual $H O X$ genes in glioblastoma behavior has been largely unknown. In particular, HOXA10 expression was recently shown to be regulated by the Trithorax protein mixed lineage leukemia (MLL), resulting in the activation of HOXA10-target genes that contribute to the tumorigenic potential of glioblastoma stem cells [14]. Another study suggested HOXA10 may contribute to temozolomide resistance in vitro [15]. While HOXA9 was also previously shown to display pro-viability and anti-apoptotic functions in glioblastoma in vitro [16], the downstream functional consequences of HOXA9 activation have remained unknown. Here, we specifically investigated for the first time the genome-wide transcriptional targets of HOXA9 in four independent GBM cell models, and evaluated how HOXA9 affects critical cellular and molecular processes of the malignant phenotype of gliomas, using a combination of in vitro and in vivo models.

Interestingly, our expression array data showed an HOXA9-mediated enrichment of genes involved in several important hallmarks of cancer (Figure 2 and Supplementary Figure 3). The fact that HOXA9 transcriptomes were so distinct within GBM cells (U87MG, U251 and GBML18) and in immortalized astrocytes (hTERT/E6/E7) cells suggests that HOXA9 targets different genes in a cell-type dependent manner, which is in accordance with previous reports in leukemia models [19]. Despite these specificities, GSEA analysis revealed that $H O X A 9$ transcriptomes are enriched for genes involved in stem cell signatures, which fits well with the previously reported $H O X$-dominated gene expression signatures found in glioblastoma stem cells [12-14, 19], and suggests HOXA9 as a critical component of that signature. Concordantly, the single overexpression of HOXA9 increased the capacity of these cells to grow as neurospheres and upregulated the expression of Nestin (Figure 3), both of which have been related to the stemness potential of GBM cells [25]. Our data in GBM also identified novel HOXA9 target genes involved in cell proliferation and cytoskeletal organization pathways, which have previously been identified as HOXA9 downstream effectors in leukemia [19]. Genes involved in other critical pathways, including DNA repair, cell invasion, cell mobility and immune response, were also found up-regulated due to HOXA9 (Figure 2, Supplementary Figure 3 and Supplementary Figure 10). Together, our findings suggest that several molecular pathways act downstream of HOXA9 to maintain a malignant phenotype in GBM. Critically, these HOXA9driven signatures may be pharmacological reverted by $\mathrm{PI} 3 \mathrm{~K}$ inhibitors and chromatin-remodeling drugs, as suggested previously $[13,16,33]$ and now by our connectivity map analyses (Supplementary Figure $3 \mathrm{H}$ ).

The importance of the cancer stem cell population in tumor initiation, progression, and response to therapy has been well documented [34]. Consistent with the increased stem cell features displayed by $H O X A 9$-positive cells, our subcutaneous xenograft models using U87MG cells showed that $H O X A 9$ accelerates tumor growth and tumor-related angiogenesis, with a concomitant increase in the expression of proteins involved in these cellular processes (Cyclin D1, Ki-67, BCL2, and PECAM1; Figure 4). Strikingly, we also showed that hTERT/E6/E7 immortalized astrocytes that overexpress $H O X A 9$ are able to originate orthotopic gliomas in vivo, in contrast to HOXA9-negative hTERT/ E6/E7 cells (Figure 5), which may partly be explained by the notion that tumor-initiating cells have high stem cell potential. While HOXA9 is an established oncogene in the context of leukemia $[35,36]$, our study is the first to clearly demonstrate the oncogenic potential of HOXA9 in gliomas.

Previous studies have provided circumstantial evidence suggesting $H O X$ genes may be important in the aggressiveness of GBM [11-13, 16]. However, the functional mechanisms by which particular $H O X$ genes may contribute to this malignant phenotype have not been elucidated. In the present study, we employed both overexpression and shRNA-mediated silencing approaches to investigate the functional roles of HOXA9 in four independent GBM models. Globally, our data show that HOXA9 increases cell viability, invasion, and resistance to cell death in GBM (Figure 6 and Supplementary Figure 11), establishing a good parallel with HOXA9 functions observed in leukemia models [37, 38]. In addition, HOXA9 significantly increased the resistance of all tested GBM cell lines to temozolomide treatment (Figure 6 and Supplementary Figure 11), establishing HOXA9 as a new biomarker of temozolomide response in GBM. Of note, this HOXA9-driven resistance to temozolomide is likely to be independent of $p 53$ function, as the cell lines tested here have different p53 status ( $p 53$ wildtype in U87MG; p53 mutant in U251; p53 inactivated in hTERT/E6/E7). Critically, the increased resistance to temozolomide mediated by HOXA9 was validated in vivo, as U87MG-HOXA9-xenografted animals presented a significantly shorter survival than those xenografted with U87MG-MSCV cells (Figure 7). In order to provide further insights into the mechanisms of HOXA9-mediated resistance to chemotherapy, which are critical to help designing novel therapeutic strategies to treat GBM patients, we investigated canonical markers 
of temozolomide response in GBM. Interestingly, HOXA9 did not affect the protein levels of MGMT, or the DNA methylation status of the MGMT promoter, which is a major regulator of temozolomide response. In contrast, HOXA9 affected the expression of other key proteins involved in temozolomide response, including down-regulation of MMR family proteins PMS2 and MSH6 (Figure 7). MMR deficiency has been implicated in preclinical studies as an additional mechanism of resistance to alkylating agents, including temozolomide [39]. Specifically, reduction of PMS2 protein levels was found to be associated with temozolomide resistance, and inactivating mutations of MSH6 gene and loss of MSH6 function have been associated with emergent temozolomide resistance in GBM patients [40, 41]. Our data suggest a novel HOXA9-driven mechanism of MMR deficiency in GBM that may decrease the therapeutic value of temozolomide. Since reestablishing MMR protein levels/function is not clinically feasible, we explored additional mechanisms by which HOXA9 may drive temozolomide resistance in GBM. Interestingly, we found that HOXA9 increases the expression of the anti-apoptotic protein BCL2 in vitro and in vivo (microarray, qPCR, immunohistochemistry and Western blot data). Importantly, the majority of GBM patients with HOXA9-high tumors also display high levels of $B C L 2$ (TCGA data), indicating that this molecular link is clinically relevant and not only exclusive of GBM models. Indeed, BCL2 was recently shown to be a target of HOXA9 in acute myeloid leukemia [42]. BCL2 has been considered an attractive target for anticancer therapies as it can be pharmacologically inhibited and, thus, potentiate apoptosis in cancer cells [30-32]. Indeed, the blockade of BCL2 activity has shown promising results in several cancers, such as pediatric lymphoblastic leukemia [43], neuroblastoma [44], melanoma [45], genitourinary neoplasm [30], and glioblastoma [46]. A recent study showed that the down-regulation of BCL2 restored temozolomide sensitization in a GBM cell line [47]. Another study showed a positive effect of ABT737, a BCL2 inhibitor, in restoring chemosensitivity to vincristine and etoposide [46]. These data prompted us to evaluate how inhibition of BCL2 function could sensitize HOXA9-positive GBM cells to temozolomide. Interestingly, a combination of temozolomide and ABT-737 had a significant increased cytotoxic effect as compared to temozolomide alone, exclusively in U87MGHOXA9 cells (Figure 7). Of note, a similar combination of temozolomide and ABT-737 did not affect the viability of BCL2-negative cells, providing a proof-of-concept that the HOXA9-mediated resistance to temozolomide can be partly reverted by specifically inhibiting BCL2 function. Of note, ABT-737 may be too large to cross the bloodbrain barrier, which could limit its clinical application. Nevertheless, malignant gliomas frequently present a focal breach of the BBB caused by a loss of tight junctions
[48], allowing blood-borne molecules to enter the brain parenchyma. On other hand, the intracranial delivery of ABT-737 might be possible in the future through the use of nanoparticle-based delivery systems, through intraventricular administration, or by using implantable wafers. Future studies should further investigate how these combinatory treatments can be more effective to treat the subset of GBM patients with high levels of HOXA9/ BCL2, helping to improve the outcome of these highly therapy resistant tumors.

In conclusion, our study establishes HOXA9 as a critical oncogene in the initiation and progression of glioma, and provides evidences into the mechanisms by which patients with HOXA9-positive GBMs respond poorly to temozolomide and have worse clinical outcomes. These findings may have clinical impact by allowing the timely identification of patients that may not benefit from temozolomide-based treatment, who could be redirected to alternative therapeutic regimens, namely based on BCL2 inhibition.

\section{MATERIALS AND METHODS}

\author{
Detailed methodologies are described in \\ Supplementary Data.
}

\section{TCGA and REMBRANDT meta-analysis in glioma patients}

Gene expression, copy number, DNA methylation, MGMT promoter methylation, GBM subtype classification, and clinical data from GBM, lower-grade glioma (LGG, WHO grades II and III), and normal samples were collected from TCGA (http://tcga-data.nci. nih.gov/). HOXA9 expression and prognostic value was also assessed in patients from REMBRANDT (https:// caintegrator.nci.nih.gov/rembrandt/).

\section{Cell lines}

Four different GBM cell line models were used: two established human GBM cell lines (U87MG and U251), immortalized human astrocytes (hTERT/E6/ E7), and a primary GBM culture (GBML18) established in our lab as previously described [49]. All cell lines were maintained in Dulbecco's Modified Eagle Medium (DMEM) supplemented with $10 \%$ FBS and $1 \%$ penicillin-streptomycin and incubated at $37^{\circ} \mathrm{C}$ in $5 \%$ $\mathrm{CO}_{2}$. The commercial cell lines U87MG and U251 were authenticated by short tandem repeat profiling.

\section{HOXA9-overexpression and silencing in GBM cells}

U87MG and hTERT/E6/E7 were previously retrovirally infected with MSCVneo vectors containing $H O X A 9$ cDNA [16]. For HOXA9 silencing, GBML18 and 
U251 cells were transfected with pGFP-V-RS plasmid containing HOXA9-specific shRNAs or non-effective shRNAs sequences (Origene).

\section{Gene expression microarray analyses}

Genome-wide transcriptome profiles of U87MG and hTERT/E6/E7 cells with MSCV (control) or HOXA9 vectors, and U251 and GBML18 with pGFPV-RS plasmid containing HOXA9-specific shRNAs or non-effective shRNAs sequences were performed by expression microarrays (Whole Human Genome 44k Oligo Microarray Kit, Agilent) and validated by RT-PCR or quantitative real-time PCR. Microarray data and full details on the experimental procedures are published online at Gene Expression Omnibus (http://www.ncbi.nlm.nih.gov/ geo/query/acc.cgi?token=ebmfagkwxrkndqx\&acc; GEO accession number GSE56517).

Differentially-expressed genes were used for Database for Annotation, Visualization and Integrated Discovery (DAVID) analyses and displayed in KEGG, GO, or Reactome pathways. For gene set enrichment analysis (GSEA; http://www.broad.mit.edu/gsea/), databases from MSigDB $\mathrm{C} 2$ collection version three were used, and results with false discovery rate (FDR) $<0.25$ were considered significant [50]. Connectivity Map was used to verify the similarity between HOXA9 transcriptome in GBM models with drug treatmentsinduced gene expression signatures in cancer models [51].

\section{Functional assays}

Neurospheres formation assay was performed in hTERT/E6/E7 and U87MG cell lines. For all cell lines the half maximal inhibitory concentration $\left(\mathrm{IC}_{50}\right)$ of temozolomide (TMZ) was determined by MTS (Promega), following the manufacturer's instructions. Time-course cell viability assays were determined by Trypan blue exclusion assays $\left(\right.$ Gibco $\left.^{\circledR}\right)$ and MTS tests. Cell death was evaluated by annexin V staining (BD Biosciences), followed by flow cytometry analyses. Invasion was measured using the BD BioCoat ${ }^{\mathrm{TM}}$ Tumor Invasion System (BD Biosciences) as indicated by the manufacturer's instructions.

\section{Immunohistochemistry, immunofluorescence and western blot}

Immunohistochemistry or immunofluorescence staining was conducted using antibodies against Cyclin D1, Ki-67, BCL2, Nestin and PECAM1. Tumor vascular area was calculated as the percentage of PECAM1 positive cells in at least 17 consecutive areas (100x: $\sim 0.63 \mathrm{~mm}^{2}$ ) evaluated per tumor. Western blot was performed using antibodies against MGMT, BCL2, MSH3, MSH2, PMS2, MSH6, MLH3, MLH1, PCNA, PARP1, APEX1, XRCC1. All antibodies are listed in Supplementary Table 2.

\section{RT-PCR and quantitative real-time PCR}

RNA extraction was performed by TRIzol (Invitrogen) and cDNA synthetized using RT-Phusion Kit, Thermo Scientific. Gene-specific mRNA levels were assessed by standard RT-PCR or quantitative PCR (qPCR), using the $\Delta \Delta \mathrm{Ct}$ method as described previously [52]. All primers sequences are listed in Supplementary Table 3.

\section{DNA isolation, bisulfite treatment and $M G M T$ methylation-specific PCR}

DNA was isolated from cell lines using TRIzol (Invitrogen) and treated with sodium bisulfite using the EZ DNA Methylation-Gold ${ }^{\mathrm{TM}}$ Kit (Zymo Research), according to the manufacturer's protocol. Methylationspecific PCR for $M G M T$ promoter was performed as previously described [53].

\section{In vivo GBM xenografts}

All experiments with mice were approved by institutional and national ethical committees (Direção Geral de Alimentação e Veterinária, Portugal) and in accordance with European Union Directive 2010/63/ EU. Subcutaneous and orthotopic xenograft models were performed by injecting U87MG and hTERT/E6/E7 cells with or without HOXA9.

\section{Statistical analyses}

SPSS 19.0 software (SPSS, Inc.), GraphPad Prism software (version 5.0) and the Bioconductor platform were used. Statistical significance was considered when $p<0.05$.

\section{ACKNOWLEDGMENTS}

The authors would like to acknowledge the funding agencies that supported this work: Fundação para a Ciência e Tecnologia (PTDC/SAU-GMG/113795/2009 and SFRH/ BPD/33612/2009 to B.M.C.; SFRH/BD/81042/2011 to M.P.; SFRH/BD/88220/2012 to A.X.M.; PTDC/SAUGMG/113795/2009 to A.I.O. and C.S.G.), Fundação Calouste Gulbenkian (B.M.C.), and Liga Portuguesa Contra o Cancro (B.M.C.), and Schering-Plough Farma (R.M.R), Portugal. Project co-financed by Programa Operacional Regional do Norte (ON.2-O Novo Norte), Quadro de Referência Estratégico Nacional (QREN), Fundo Europeu de Desenvolvimento Regional (FEDER). The authors would like to extend their appreciation to Dr. Chris Jones and Dr. Sergey Popov (ICR, UK) for helpful assistance regarding histopathological analysis of xenograft tumors, Dr. Russell Pieper for sharing the hTERT/E6/E7 cell line (UCSF, USA), and Dr. Joseph Costello (UCSF, USA) for critical review of the manuscript. 


\section{Conflict of interests}

The authors disclose any potential conflict of interest.

\section{REFERENCES}

1. Ohgaki H, Kleihues P. Genetic pathways to primary and secondary glioblastoma. Am J Pathol. 2007; 170:1445-1453.

2. CBTRUS. Primary brain and other nervous system tumors, estimated number of cases overall and by behavior by state, 2012; Primary malignant brain and other nervous system tumors, estimated number of deaths by state. 2012. http:// www.cbtrus.org/2007-2008/2007-2008.

3. Burnet NG, Jefferies SJ, Benson RJ, Hunt DP, Treasure FP. Years of life lost (YLL) from cancer is an important measure of population burden-and should be considered when allocating research funds. Br J Cancer. 2005; 92:241-245.

4. Stupp R, Mason WP, van den Bent MJ, Weller M, Fisher B, Taphoorn MJ, Belanger K, Brandes AA, Marosi C, Bogdahn U, Curschmann J, Janzer RC, Ludwin SK, Gorlia T, Allgeier A, Lacombe D, et al. Radiotherapy plus concomitant and adjuvant temozolomide for glioblastoma. N Engl J Med. 2005; 352:987-996.

5. Stupp R, Hegi ME, Mason WP, van den Bent MJ, Taphoorn MJ, Janzer RC, Ludwin SK, Allgeier A, Fisher B, Belanger K, Hau P, Brandes AA, Gijtenbeek J, Marosi C, Vecht CJ, Mokhtari K, et al. Effects of radiotherapy with concomitant and adjuvant temozolomide versus radiotherapy alone on survival in glioblastoma in a randomised phase III study: 5-year analysis of the EORTC-NCIC trial. Lancet Oncol. 2009; 10:459-466.

6. Hegi ME, Diserens AC, Gorlia $\mathrm{T}$, Hamou MF, de Tribolet N, Weller M, Kros JM, Hainfellner JA, Mason W, Mariani L, Bromberg JE, Hau P, Mirimanoff RO, Cairncross JG, Janzer RC, Stupp R. MGMT gene silencing and benefit from temozolomide in glioblastoma. N Eng $\mathrm{J}$ Med. 2005; 352:997-1003.

7. Olson RA, Brastianos PK, Palma DA. Prognostic and predictive value of epigenetic silencing of MGMT in patients with high grade gliomas: a systematic review and metaanalysis. J Neurooncol. 2011; 105:325-335.

8. Raman V, Martensen SA, Reisman D, Evron E, Odenwald WF, Jaffee E, Marks J, Sukumar S. Compromised HOXA5 function can limit p53 expression in human breast tumours. Nature. 2000; 405:974-978.

9. Care A, Felicetti F, Meccia E, Bottero L, Parenza M, Stoppacciaro A, Peschle C, Colombo MP. HOXB7: a key factor for tumor-associated angiogenic switch. Cancer Res. 2001; 61:6532-6539.

10. Shah N, Sukumar S. The hox genes and their roles in oncogenesis. Nat Rev Cancer. 2010; 10:361-371.
11. Abdel-Fattah R, Xiao A, Bomgardner D, Pease CS, Lopes MB, Hussaini IM. Differential expression of HOX genes in neoplastic and non-neoplastic human astrocytes. The Journal of pathology. 2006; 209:15-24.

12. Murat A, Migliavacca E, Gorlia T, Lambiv WL, Shay T, Hamou MF, de Tribolet N, Regli L, Wick W, Kouwenhoven MC, Hainfellner JA, Heppner FL, Dietrich PY, Zimmer Y, Cairncross JG, Janzer RC, et al. Stem cell-related "self-renewal" signature and high epidermal growth factor receptor expression associated with resistance to concomitant chemoradiotherapy in glioblastoma. J Clin Oncol. 2008; 26:3015-3024.

13. Gaspar N, Marshall L, Perryman L, Bax DA, Little SE, Viana-Pereira M, Sharp SY, Vassal G, Pearson AD, Reis RM, Hargrave D, Workman P, Jones C. MGMTindependent temozolomide resistance in pediatric glioblastoma cells associated with a PI3-kinase-mediated HOX/ stem cell gene signature. Cancer Res. 2010; 70:9243-9252.

14. Gallo M, Ho J, Coutinho FJ, Vanner R, Lee L, Head R, Ling EK, Clarke ID, Dirks PB. A tumorigenic MLLhomeobox network in human glioblastoma stem cells. Cancer Res. 2013; 73:417-427.

15. Kim JW, Kim JY, Kim JE, Kim SK, Chung HT, Park CK. HOXA10 is associated with temozolomide resistance through regulation of the homologous recombinant DNA repair pathway in glioblastoma cell lines. Genes Cancer. 2014; 5:165-174.

16. Costa BM, Smith JS, Chen Y, Chen J, Phillips HS, Aldape KD, Zardo G, Nigro J, James CD, Fridlyand J, Reis RM, Costello JF. Reversing HOXA9 oncogene activation by PI3K inhibition: epigenetic mechanism and prognostic significance in human glioblastoma. Cancer Res. 2010; 70:453-462.

17. TCGA: Comprehensive genomic characterization defines human glioblastoma genes and core pathway. Nature. 2008; 455:1061-1068.

18. Verhaak RG, Hoadley KA, Purdom E, Wang V, Qi Y, Wilkerson MD, Miller CR, Ding L, Golub T, Mesirov JP, Alexe G, Lawrence M, O'Kelly M, Tamayo P, Weir BA, Gabriel S, et al. Integrated genomic analysis identifies clinically relevant subtypes of glioblastoma characterized by abnormalities in PDGFRA, IDH1, EGFR, and NF1. Cancer Cell. 2010; 17:98-110.

19. Dorsam ST, Ferrell CM, Dorsam GP, Derynck MK, Vijapurkar U, Khodabakhsh D, Pau B, Bernstein H, Haqq CM, Largman C, Lawrence HJ. The transcriptome of the leukemogenic homeoprotein HOXA9 in human hematopoietic cells. Blood. 2004; 103:1676-1684.

20. Zhu VF, Yang J, Lebrun DG, Li M. Understanding the role of cytokines in glioblastoma multiforme pathogenesis. Cancer Lett. 2012; 316:139-150.

21. Yoshida M, Kijima M, Akita M, Beppu T. Potent and specific inhibition of mammalian histone deacetylase both in vivo and in vitro by trichostatin A. The Journal of biological chemistry. 1990; 265:17174-17179. 
22. Yamashita Y, Shimada M, Harimoto N, Rikimaru T, Shirabe K, Tanaka S, Sugimachi K. Histone deacetylase inhibitor trichostatin A induces cell-cycle arrest/apoptosis and hepatocyte differentiation in human hepatoma cells. Int J Cancer. 2003; 103:572-576.

23. Sauvageot CM, Weatherbee JL, Kesari S, Winters SE, Barnes J, Dellagatta J, Ramakrishna NR, Stiles CD, Kung AL, Kieran MW, Wen PY. Efficacy of the HSP90 inhibitor 17-AAG in human glioma cell lines and tumorigenic glioma stem cells. Neuro Oncol. 2009; 11:109-121.

24. Fukuyo Y, Hunt CR, Horikoshi N. Geldanamycin and its anti-cancer activities. Cancer Lett. 2010; 290:24-35.

25. Singh SK, Clarke ID, Terasaki M, Bonn VE, Hawkins C, Squire J, Dirks PB. Identification of a cancer stem cell in human brain tumors. Cancer Res. 2003; 63:5821-5828.

26. Singh SK, Clarke ID, Hide T, Dirks PB. Cancer stem cells in nervous system tumors. Oncogene. 2004; 23:7267-7273.

27. Dirks PB. Brain tumor stem cells: the cancer stem cell hypothesis writ large. Mol Oncol. 2010; 4:420-430.

28. Sonoda Y, Ozawa T, Hirose Y, Aldape KD, McMahon M, Berger MS, Pieper RO. Formation of intracranial tumors by genetically modified human astrocytes defines four pathways critical in the development of human anaplastic astrocytoma. Cancer Res. 2001; 61:4956-4960.

29. Liu L, Gerson SL. Targeted modulation of MGMT: clinical implications. Clin Cancer Res. 2006; 12:328-331.

30. Hall C, Troutman SM, Price DK, Figg WD, Kang MH. Bcl-2 family of proteins as therapeutic targets in genitourinary neoplasms. Clin Genitourin Canc. 2013; 11:10-19.

31. Oltersdorf T, Elmore SW, Shoemaker AR, Armstrong RC, Augeri DJ, Belli BA, Bruncko M, Deckwerth TL, Dinges J, Hajduk PJ, Joseph MK, Kitada S, Korsmeyer SJ, Kunzer AR, Letai A, Li C, et al. An inhibitor of Bcl-2 family proteins induces regression of solid tumours. Nature. 2005; 435:677-681.

32. Souers AJ, Leverson JD, Boghaert ER, Ackler SL, Catron ND, Chen J, Dayton BD, Ding H, Enschede SH, Fairbrother WJ, Huang DC, Hymowitz SG, Jin S, Khaw SL, Kovar PJ, Lam LT, et al. ABT-199, a potent and selective BCL-2 inhibitor, achieves antitumor activity while sparing platelets. Nat Med. 2013; 19:202-208.

33. Cha TL, Zhou BP, Xia W, Wu Y, Yang CC, Chen CT, Ping B, Otte AP, Hung MC. Akt-mediated phosphorylation of EZH2 suppresses methylation of lysine 27 in histone H3. Science. 2005; 310:306-310.

34. Nguyen LV, Vanner R, Dirks P, Eaves CJ. Cancer stem cells: an evolving concept. Nat Rev Cancer. 2012; 12:133-143.

35. Kroon E, Thorsteinsdottir U, Mayotte N, Nakamura T, Sauvageau G. NUP98-HOXA9 expression in hemopoietic stem cells induces chronic and acute myeloid leukemias in mice. EMBO J. 2001; 20:350-361.
36. Ayton PM, Cleary ML. Transformation of myeloid progenitors by MLL oncoproteins is dependent on Hoxa7 and Hoxa9. Genes Dev. 2003; 17:2298-2307.

37. Smith LL, Yeung J, Zeisig BB, Popov N, Huijbers I, Barnes J, Wilson AJ, Taskesen E, Delwel R, Gil J, Van Lohuizen M, So CW. Functional crosstalk between Bmil and MLL/Hoxa9 axis in establishment of normal hematopoietic and leukemic stem cells. Cell Stem Cell. 2011; 8:649-662.

38. Gibbs KD, Jager A, Crespo O, Goltsev Y, Trejo A, Richard CE, Nolan GP. Decoupling of tumor-initiating activity from stable immunophenotype in HoxA9-Meis1driven, AML. Cell Stem Cell. 2012; 10:210-217.

39. Cahill DP, Levine KK, Betensky RA, Codd PJ, Romany CA, Reavie LB, Batchelor TT, Futreal PA, Stratton MR, Curry WT, Iafrate AJ, Louis DN. Loss of the mismatch repair protein MSH6 in human glioblastomas is associated with tumor progression during temozolomide treatment. Clin Cancer Res. 2007; 13:2038-2045.

40. Hunter C, Smith R, Cahill DP, Stephens P, Stevens C, Teague J, Greenman C, Edkins S, Bignell G, Davies H, O'Meara S, Parker A, Avis T, Barthorpe S, Brackenbury L, Buck $\mathrm{G}$, et al. A hypermutation phenotype and somatic MSH6 mutations in recurrent human malignant gliomas after alkylator chemotherapy. Cancer Res. 2006; 66:3987-3991.

41. Yip S, Miao J, Cahill DP, Iafrate AJ, Aldape K, Nutt CL, Louis DN. MSH6 mutations arise in glioblastomas during temozolomide therapy and mediate temozolomide resistance. Clin Cancer Res. 2009; 15:4622-4629.

42. Brumatti G, Salmanidis $\mathrm{M}$, Kok $\mathrm{CH}$, Bilardi RA, Sandow JJ, Silke N, Mason K, Visser J, Jabbour AM, Glaser SP, Okamoto T, Bouillet P, D'Andrea RJ, Ekert PG. HoxA9 regulated $\mathrm{Bcl}-2$ expression mediates survival of myeloid progenitors and the severity of HoxA9-dependent leukemia. Oncotarget. 2013; 4:1933-1947.

43. Szymanska B, Wilczynska-Kalak U, Kang MH, Liem NL, Carol H, Boehm I, Groepper D, Reynolds CP, Stewart CF, Lock RB. Pharmacokinetic modeling of an induction regimen for in vivo combined testing of novel drugs against pediatric acute lymphoblastic leukemia xenografts. PLoS One. 2012; 7:e33894.

44. Fang H, Harned TM, Kalous O, Maldonado V, DeClerck YA, Reynolds CP. Synergistic activity of fenretinide and the Bcl-2 family protein inhibitor ABT-737 against human neuroblastoma. Clin Cancer Res. 2011; 17:7093-7104.

45. Reuland SN, Goldstein NB, Partyka KA, Cooper DA, Fujita M, Norris DA, Shellman YG. The combination of BH3-mimetic ABT-737 with the alkylating agent temozolomide induces strong synergistic killing of melanoma cells independent of p53. PLoS One. 2011; 6:e24294.

46. Tagscherer KE, Fassl A, Campos B, Farhadi M, Kraemer A, Bock BC, Macher-Goeppinger S, Radlwimmer B, Wiestler OD, Herold-Mende C, Roth W. Apoptosis-based treatment of glioblastomas with ABT-737, a novel small molecule inhibitor of Bcl-2 family proteins. Oncogene. 2008; 27:6646-6656. 
47. Qi XC, Xie DJ, Yan QF, Wang YR, Zhu YX, Qian C, Yang SX. LRIG1 dictates the chemo-sensitivity of temozolomide (TMZ) in U251 glioblastoma cells via down-regulation of EGFR/topoisomerase-2/Bcl-2. Biochem Biophys Res Commun. 2013; 437:565-572.

48. Watkins S, Robel S, Kimbrough IF, Robert SM, EllisDavies G, Sontheimer H. Disruption of astrocyte-vascular coupling and the blood-brain barrier by invading glioma cells. Nat Commun. 2014; 5:4196.

49. Azari H, Millette S, Ansari S, Rahman M, Deleyrolle LP, Reynolds BA. Isolation and expansion of human glioblastoma multiforme tumor cells using the neurosphere assay. J Vis Exp. 2011; 56:e3633.

50. Subramanian A, Tamayo P, Mootha VK, Mukherjee S, Ebert BL, Gillette MA, Paulovich A, Pomeroy SL, Golub TR, Lander ES, Mesirov JP. Gene set enrichment analysis: a knowledgebased approach for interpreting genome-wide expression profiles. Proc Natl Acad Sci U S A. 2005; 102:15545-15550.
51. Lamb J, Crawford ED, Peck D, Modell JW, Blat IC, Wrobel MJ, Lerner J, Brunet JP, Subramanian A, Ross KN, Reich M, Hieronymus H, Wei G, Armstrong SA, Haggarty SJ, Clemons PA, et al. The connectivity map: using gene-expression signatures to connect small molecules, genes, and disease. Science. 2006; 313:1929-1935.

52. Livak KJ, Schmittgen TD. Analysis of relative gene expression data using real-time quantitative PCR and the 2(-Delta Delta C(T)) method. Methods. 2001; 25:402-408.

53. Costa BM, Caeiro C, Guimaraes I, Martinho O, Jaraquemada T, Augusto I, Castro L, Osorio L, Linhares P, Honavar M, Resende M, Braga F, Silva A, Pardal F, Amorim J, Nabico R, et al. Prognostic value of MGMT promoter methylation in glioblastoma patients treated with temozolomide-based chemoradiation: a Portuguese multicentre study. Oncol Rep. 2010; 23:1655-1662. 